\title{
Benefits and Limitations of Common Directional Microphones in Real-World Sounds
}

\author{
Xubao Zhang ${ }^{1,2}$ \\ ${ }^{1}$ Research \& Development, Sonova Unitron, Oticon, Canada \\ ${ }^{2}$ Electronic Engineering Department, Xi' an Electronic Science and Technology University, Xi'an, China
}

Email address:

xbzwdl@yahoo.com

\section{To cite this article:}

Xubao Zhang. Benefits and Limitations of Common Directional Microphones in Real-World Sounds. Clinical Medicine Research. Vol. 7, No. 5, 2018, pp. 103-118. doi: 10.11648/j.cmr.20180705.12

Received: September 6, 2018; Accepted: September 26, 2018; Published: October 26, 2018

\begin{abstract}
We present extensive experimental data to objectively evaluate the benefits and limitations of common directional microphones in real-world sound fields. The microphones include a conventional directional microphone(DM), a balanced $\mathrm{DM}$, etc., plus the Omni microphone (mic) as a benchmark. The evaluation focuses on noise outputs, signal-to-noise $\operatorname{ratios}(\mathrm{S} / \mathrm{Ns})$ and distortions; the real-world sounds include male voices, female voices, babble noises, white noises and talking interferences. Each type of noises is at 4 or 5 levels, from 30 to $70 \mathrm{~dB}$ SPL, at $10 \mathrm{~dB}$ step, and each talking interference is at 3 levels: 50, 60 and $70 \mathrm{~dB}$ SPL. The research methods include analytically deriving sensitivity-gains, statistically calculating the three mics' outputs, experimentally viewing waveforms and spectra, and using large-sample wave files for a high confidence level. According to the experimental results, this paper concludes that 1) for a conversation in a quiet field, in soft or low noise field, the common DMs achieve comfortable S/Ns: 7 to $33 \mathrm{~dB}$, similar to what the Omni mic does; 2) for a conversation in low, competing or strong talking interference fields, the common DMs achieve about $16 \mathrm{~dB}$ better $\mathrm{S} / \mathrm{N}$ than the Omni mic does; 3 ) for a conversation in competing or strong surrounding noise field, the common DMs do not achieve beneficial $\mathrm{S} / \mathrm{N}$ to understand speech; the common DMs' noises are close to the Omni mic noise; 4) in various experiments, the balanced DM preserve speech fidelity well as the Omni mic does, while the conventional DM does poorly. This paper further introduces the Simulink experimental manipulations, such as digital FIR filters' design, stereo channels' wave files creation, etc., in the Appendix.
\end{abstract}

Keywords: Directional Microphone, Speech Enhancement, Noise Suppression, Distortion and Simulink

\section{Introduction}

Directional microphones (DMs) in hearing aids have been researched and developed for more than 20 years. While the noise suppression benefits for hearing-impaired persons are established, the limitations have not been popularly known. At present, the DMs have not been researched thoroughly [1]. Audiologists and hearing professionals have been developing new DM technologies. The SpeechFocus can provide an adaptive beam-former [2]. When the speech sound comes from the back, on the left or right site, the lobe of the beam-former faces the back, the left or right side. A technology similar to the SpeechFocus, called auto ZoomControl, earlier was proposed [3, 4]; further, when it is combined with a binaural wireless communication, the auto
ZoomControl is nearly optimized. A super-directional beamformer is able to increase $\mathrm{S} / \mathrm{N}$ to achieve normal speech understanding in noise [5]; this beam-former can form a proper width beam so as to attenuate off-beam signals and to preserve spatial cues of the environment. Through six different experiments, the conclusion was that this beamformer outperformed the Omni mic at noisiness and acceptance, but specific conditions of the experimental noise sources were not described. These new technologies were upgraded and approximated adaptive beam-formers, thus offering $\mathrm{S} / \mathrm{N}$ improvement and better speech intelligibility. Hopefully, these new technologies will be implemented in available products in near future.

However, audiologists, developing manufacturers and researchers never stop testing and evaluating the performance of existing directional hearing products and technologies [6- 
8]. A three-year investigation for DMs' effectiveness on 94 subjects was conducted [9], and it concluded that those directional hearing aids performed better in objective $\mathrm{S} / \mathrm{N}$ measurement in the laboratory, but advantages were less clear for subjective measurements in environments. A DM evaluation study was conducted, based on nine-article literature review [10], and it concluded that those evidences of DMs' effectiveness provided weak support; the careful consideration of assessing methodologies was encouraged. Simulink experiments using wave files of real-world voices and noises were proposed [11]. Based on experimental polar plots of a conventional DM, it was suggested that the DM can obtain much more $\mathrm{S} / \mathrm{N}$ benefit than the Omni mic for beamed noise sources, such as a talking interference, but not for a surrounding noise.

A balanced DM is a conventional DM whose frequency response is balanced by multi-band gains. In a practical directional hearing aid, balanced processing minimizes spectrum distortion. Therefore, conventional DMs and balanced DMs have been common DMs in real-life hearing aids. Here we evaluate the common DMs' performance, using the Omni mic as a benchmark, on speech enhancement, noise suppression, $\mathrm{S} / \mathrm{N}$ improvement and spectrum distortion; for a high confidence level, we used various noises and speech voices from large-sample, real-world sources.

\section{Internal Noises of DMs}

Usually, a DM is composed of two or three Omni microphones (mics) located on a line array and an operation circuit. When we measured the equivalent input noise level (EINL) of a directional hearing aid, the EINL in directional mode always was 5 7 dB higher than that in Omni mode. DM product specifications also indicate that the EINL of a DM, e.g., $32 \mathrm{~dB}$ SPL, is larger than the EINL of its Omni mics, e.g., $26.5 \mathrm{~dB}$ SPL. The evidences tell us that internal noise level of a DM is higher than that of an Omni mic.

Internal noises of the Omni mics are a critical factor affecting the DM output noise. Miniature microphones used for hearing aids are extremely refined, as required by hearing aid manufacturers. A mic is usually made up of an electret condenser sensor and an integrated circuit(IC) amplifier. Figure 1 shows an anatomy diagram of a typical electret condenser microphone [12]. The diaphragm is metalized on the outside or inside surface, which electrically conducts to a part of the mic case. The metal back-plate is coated with electret material. The diaphragm and back-plate form a parallel plate capacitor. This is why the mic output characterizes capacitance. When a signal is generated between the diaphragm and back-plate, it is delivered to gate of a field effect transistor (FET) in IC, which has a very high input impedance and low output impedance. The IC amplifier has an extremely wide, flat frequency response and very low noise [13]. Thus, the frequency response of an electret mic is dominated by the electret sensor response.

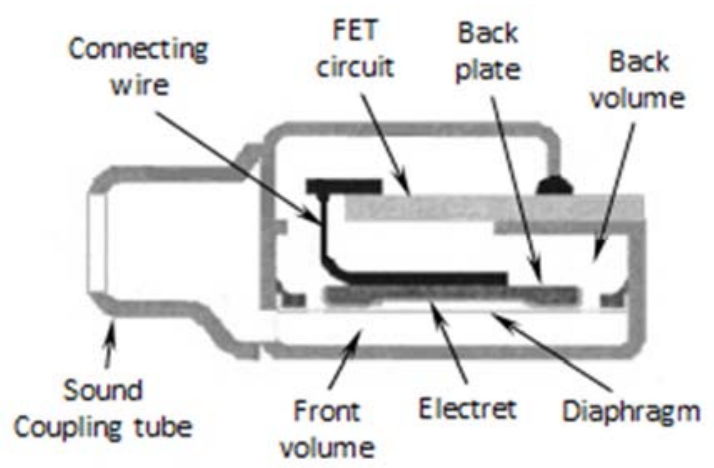

Figure 1. Anatomy of an electret condenser microphone.

Obviously, the electret mic internal noise originates from its sensor and IC amplifier. There are air flows to move along three parts of the electret sensor, the front volume, the gap between the diaphragm and back-plate, and the back volume. By physics, when molecules of the air flows impact the backplate, the diaphragm and the electret, the parts produce noises. The noises are of white spectrum because samplings of each noise output are independent over time. An evidence that the noises originate from the air flows is that the mic noise output measured in a vacuum container is significantly lower than that measured in normal air [13].

An electronic device usually makes three types of noises: thermal, shot and flicker. Thermal noise originates from a heating element, usually, a heating resister; and its spectrum is a constant, related to the element temperature and resistance. Shot noise originates only from electric current across potential barriers in the semiconductor element, so the FET in the IC is the only source of shot noise. Flicker noise is also called $1 / \mathrm{f}$ noise, its major energy distributes in a low frequency region. The former two noises in the IC are of white spectrum and dominant, then total output noise of the electret microphone is considered an approximately white noise.

Sonion manufactures various miniature acoustic devices, including hearing aid microphones with low noise levels. Sonion Data Sheets provide the "Typical response curve" and "Typical $1 / 3$ octave equivalent noise" [14]. The former is a sensitivity curve $(\mathrm{dB}$ re $1 \mathrm{~V} / \mathrm{Pa})$, and the latter is an equivalent input noise curve (dB SPL). In order to obtain a curve of microphone output noise, we first need to transform unit of the $\mathrm{Y}$ axis sensitivity into ( $\mathrm{dB}$ re $1 \mathrm{~V} / \mathrm{SPL}$ ). Since $0 \mathrm{~dB}$ SPL is equivalent to $20 \mu \mathrm{Pa}, 0 \mathrm{~dB}$ SPL is just equivalent to $-94 \mathrm{~dB}$ re $1 \mathrm{~Pa}$; in addition, $(-94 \mathrm{~dB}$ re $1 \mathrm{~V} / \mathrm{SPL})=(-34 \mathrm{~dB} \mathrm{re} 1 \mathrm{mV} / \mathrm{SPL})$. We selected three Sonion microphones, models: 6922, 6913 and 6295. A microphone output noise is equal to its equivalent input noise times its sensitivity, then the mics' output noises were calculated, as showed in Figure 2. The spectra of the output noises are not so white, caused by Flicker noise and acoustic resonance of the mics. Meanwhile, we smoothed the equivalent noise of 6922 at $10 \mathrm{k} \mathrm{Hz}$. The graphs are bar type with $1 / 3$ octave band, and their unit is (dB re. $\mathrm{mV})$. 


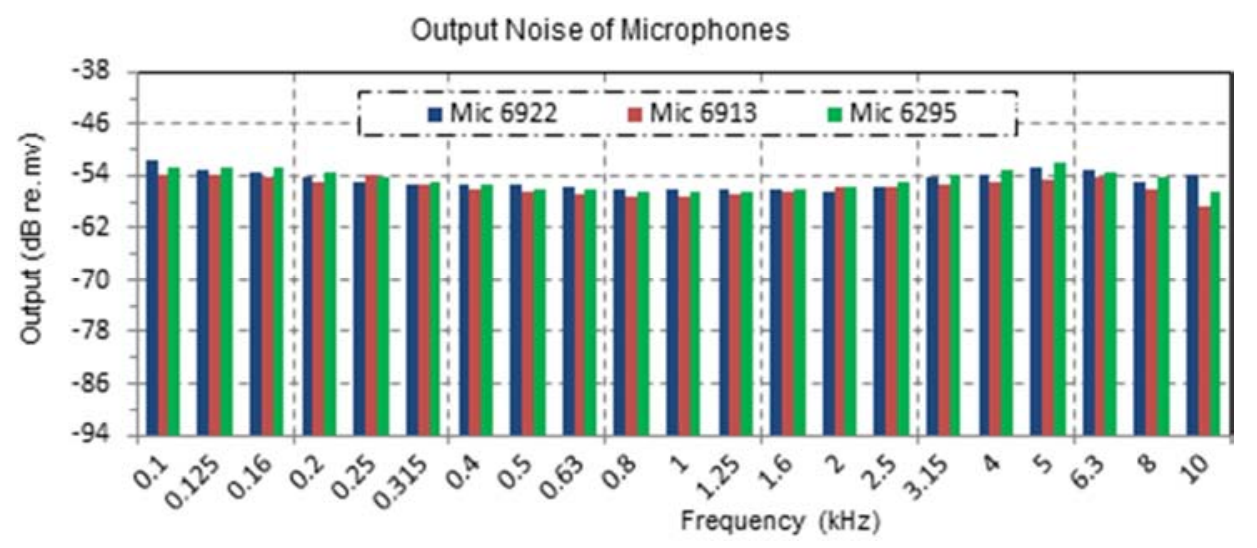

Figure 2. Output noises of three Sonion microphones.

\section{Basic Behaviors of a Few DMs}

When two Omni mics combine with a delay filter and a subtracter, as shown in Figure 3, they build a simple sound beam-former, i.e., a conventional DM, also called a 1st-order DM. In practical applications, a conventional DM has evolved into multiple varieties, such as balanced DM, 2ndorder DM, adaptive DM, etc. Practical DM circuits are complex, but their performance is still determined by their basic configurations.

\subsection{A conventional DM}

In Figure 3, the solid arrows represent the $0^{\circ}$ (front) incidence, and the dashed arrows represent the non-zero degree incidence. Without losing generality, the Omni mics' sensitivities are assigned as 1 , and the $\mathrm{A} / \mathrm{D}$ converters are ignored. In the case that incoming sounds are pure tones, assuming that the front mic output is $y_{F}(t)=\sin (2 \pi f t)$, $f$ is tone frequency and $t$ is time. The rear mic output is $y_{R}(t)=$ $\mathrm{y}_{\mathrm{F}}(\mathrm{t}-\delta(\theta))$, and $\delta(\theta)$ is external delay time between the rear mic and front mic signals. Depending on the two mic ports' spacing $d_{p}$ and incident angle $\theta, \delta(\theta)$ can be denoted as $\delta(\theta)=$ $\Delta \cos (\theta), \Delta$ is delay time of the ports' spacing. A delay filter is in the rear mic output circuit, and its parameter $\tau$ is called internal delay time, controlling the DM polar pattern shape. The DM output is the front mic output minus the filter output,

$$
\begin{aligned}
& \left.y_{D M}(t)=\sin (2 \pi f t)-\sin 2 \pi f(t-\delta(\theta)-\tau)\right] \\
& =2 \sin \pi f(\tau+\delta(\theta))] \cos 2 \pi f t-\pi f(\tau+\delta(\theta))]
\end{aligned}
$$

The DM output still is a tone signal with an additional phase $-\pi f(\tau+\delta(\theta))$ and has an amplitude $2 \sin \pi f(\tau+$ $\delta(\theta))]$. The DM spatial performance focuses on its gain polar pattern. Here, we concern only with the DM amplitude, which is related to ports' spacing, filter delay and incident angle. We still use a strict concept, sensitivity-gain(S-gain) 11]. When $\tau=\Delta$, the DM of Figure 3 is a typical cardioid DM. We can derive S-gain polar pattern of the cardioid DM from (1) as

$$
\left.\mathrm{g}_{\mathrm{CM}}(\theta, \mathrm{f})=2 \sin \pi \mathrm{f} \Delta(1+\cos (\theta))\right]
$$

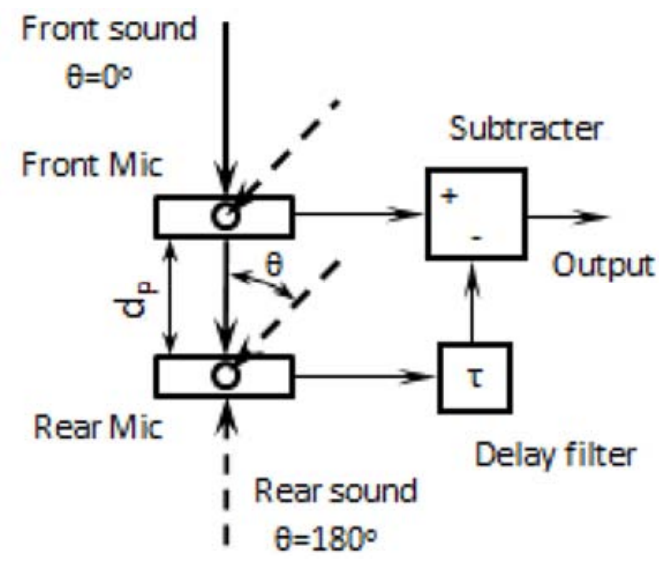

Figure 3. Basic configuration of a conventional DM.

Assuming $\mathrm{d}_{\mathrm{p}}=16 \mathrm{~mm}$ or $\Delta=0.04662 \mathrm{~ms}$, we can plot polar patterns of the cardioid DM, as shown in Figure 4. They result from three tones of frequencies: $5 \mathrm{k}, 2 \mathrm{k}$ and $500 \mathrm{~Hz}$, and each pattern has a zero notch at incident $180^{\circ}$. In all the experiments below, we used the $5 \mathrm{k} \mathrm{Hz}$ tone to represent the high frequency region; $2 \mathrm{k} \mathrm{Hz}$, mid frequency region; and 500 $\mathrm{Hz}$, low frequency region. In Figure 4, the outer pattern of $5 \mathrm{k}$ $\mathrm{Hz}$ has a max gain $2(6 \mathrm{~dB})$ at $0^{\circ}$; and the inner one of $500 \mathrm{~Hz}$ has a $0^{\circ}$ gain $0.292(-10.7 \mathrm{~dB})$. The lower the frequency, the less the gain. Only one polar pattern of $5 \mathrm{k} \mathrm{Hz}$ shown may mislead performance of the DM.

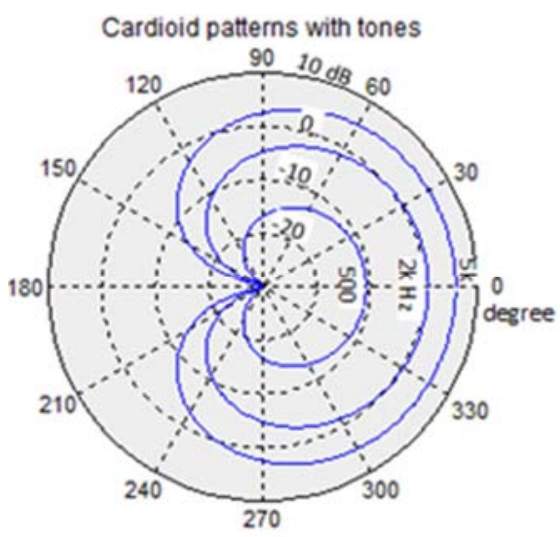

Figure 4. Polar pattern of a conventional cardioid DM. 


\subsection{A 2nd-order DM}

Based on the DM configuration shown in Figure 3, we can build a high-order sound beam-former by combining more Omni mics on a line array with more delay filters and subtracters. Figure 5 shows a 2nd-order DM configuration, which is composed of three conventional 1st-order DMs. The front mic, mid mic, subtracter1 and delay1 form a front 1storder DM. The mid mic, rear mic, subtracter2 and delay2 form a rear 1st-order DM. The output of the front DM, the output of the rear DM, subtracter3 and delay3 form a 3rd 1storder DM, and its output is just the 2nd-order DM output. Assuming that the front mic output is $\mathrm{y}_{\mathrm{F}}(\mathrm{t})=\sin (2 \pi \mathrm{ft})$, the mid mic output is $\left.\mathrm{y}_{\mathrm{M}}(\mathrm{t})=\sin 2 \pi \mathrm{f}(\mathrm{t}-\delta(\theta))\right]$, and $\tau 1=\Delta$ the delay time between front mic and mid mic ports, then output of the front 1st-order DM is

$$
\begin{aligned}
y_{D M 1}(t)= & \sin (2 \pi f t)-\sin 2 \pi f(t-\delta(\theta)-\tau 1)] \\
= & 2 \sin \pi f \Delta(1+\cos (\theta))] \\
& \cos \{2 \pi f t-\pi f \Delta+\delta(\theta)]\}
\end{aligned}
$$

When $\tau 2=\Delta$, output of the rear 1st-order DM is

$$
\begin{aligned}
& \left.\mathrm{y}_{\mathrm{DM} 2}(\mathrm{t})=\sin 2 \pi \mathrm{f}(\mathrm{t}-\Delta)\right]- \\
& \sin 2 \pi \mathrm{f}(\mathrm{t}-\Delta-\delta(\theta)-\tau 2)] \\
& =2 \sin \{\pi \mathrm{f} \Delta 1+\cos (\theta)]\} \\
& \cos \{2 \pi \mathrm{f}(\mathrm{t}-\Delta)-\pi \mathrm{f} \Delta+\delta(\theta)]\} \\
& =\mathrm{y}_{\mathrm{DM} 1}(\mathrm{t}-\Delta)
\end{aligned}
$$

Comparing equations (4) and (3), we can know that amplitude of the rear 1st-order DM output is equal to that of the front 1st-order DM output, and their time functions have a time difference $\Delta$ only. When the two output signals are used as the inputs of the 3rd 1st-order DM, the output of the 3rd 1st-order DM can be derived as

$$
\begin{aligned}
& \quad y_{D M 3}(t)=y_{D M 1}(t)-y_{D M 2}(t-\tau 3) \\
& =2 \sin \pi f \Delta(1+\cos (\theta))] 2 \sin \{\pi f \tau 3+ \\
& \quad \delta(\theta)]\} \sin \{2 \pi f(t-\Delta-\tau 3)-\pi f[\tau 3+\delta(\theta)]\}
\end{aligned}
$$

where $2 \sin \pi f \Delta(1+\cos (\theta))]$ has a cardioid pattern and $2 \sin \{\pi f[\tau 3+\delta(\theta)]\}$ has a flexible pattern, depending on the value of $\tau 3$. When $\tau 3=\Delta$, the pattern of the 2 nd-order DM is a 2nd-order cardioid, and can be plotted by means of

$$
\mathrm{g}_{\text {2осм }}(\theta, \mathrm{f})=4 \sin ^{2}\{\pi \mathrm{f} \Delta(1+\cos (\theta))\}
$$

Figure 6 shows the S-gain polar patterns of the 2nd-order cardioid DM, which result from three tones of frequencies $5 \mathrm{k}, 2 \mathrm{k}$ and $500 \mathrm{~Hz}$. Each pattern has a zero notch at incident $180^{\circ}$. The outer pattern results from the $5 \mathrm{k} \mathrm{Hz}$ tone, having a $\max$ gain $4(12 \mathrm{~dB})$ at $0^{\circ}$; the inner one, from the $500 \mathrm{~Hz}$ tone, having a gain $0.0853(-21.4 \mathrm{~dB})$ at $0^{\circ}$. Thus, the resulting pattern has a narrower lobe than the 1st-order DM.

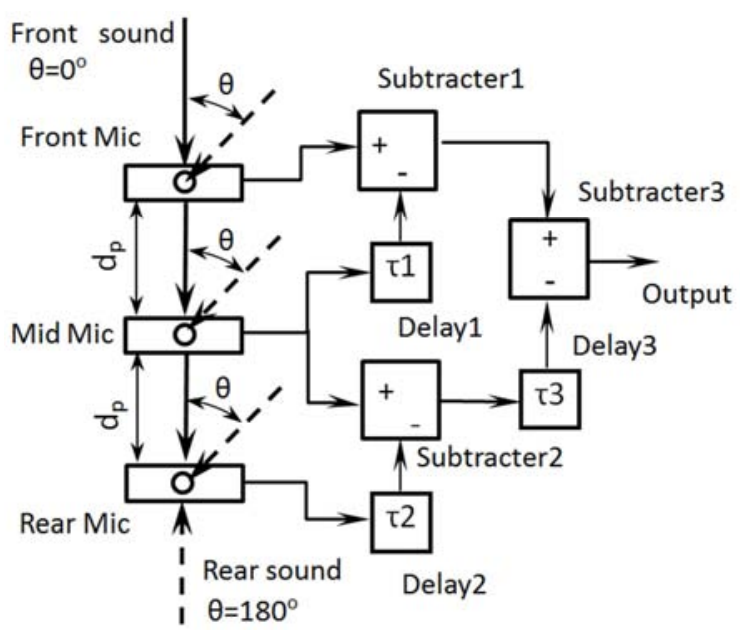

Figure 5. Basic configuration of a 2nd-order DM.

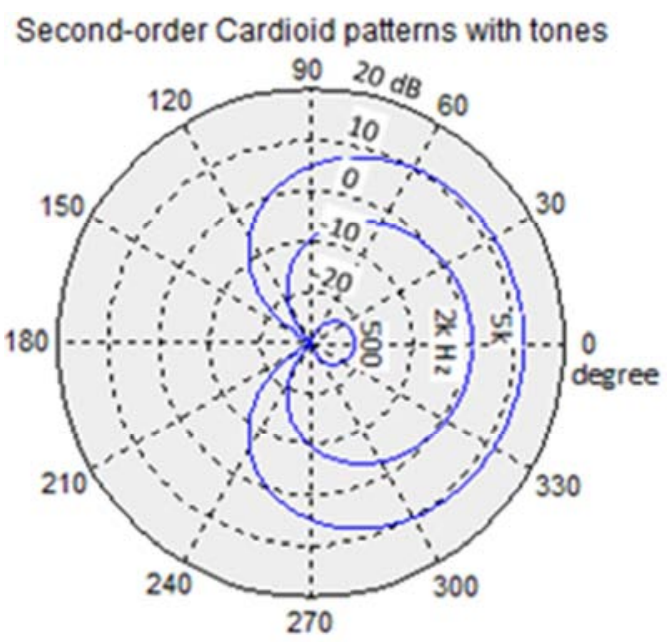

Figure 6. Polar patterns of a 2nd-order Cardioid DM.

\subsection{A Balanced DM}

Frequency response of a conventional DM has a $6 \mathrm{~dB}$ up slope in the low-mid frequency region, as shown in Figure 9. In practice, the response slope can be reduced by balancing the response with multi-band multipliers, as shown in Figure 7. We designed eight-band multipliers, each of which was composed of a band-pass filter and a multiplier. The entire coverage of the eight bands is $200 \sim 8000 \mathrm{~Hz}$, meeting the related requirements of Standards ANSI S3.22 and IEC 60118. The input in Figure 7 is connected to the conventional DM output. In a modern hearing aid, advantage features, such as noise reduction, feedback cancelation, etc., also are implemented in such multi-band processor. Thus, in a practical hearing aid, the multi-band processor functions in Omni mode too. The multiplier value of each band depends on the corresponding band gain of the conventional DM. The lower the gain is, the larger the multiplier is. Design of these band-pass filters focused on outputs' balance and delay times' consistency, which affected the speech signal fidelity. The frequency response ripples were tested within $\pm 1.2 \mathrm{~dB}$, and the delay times, about 80 samples, $1.8 \mathrm{~ms}$. For details of band-pass filter design, refer to the Appendix. 


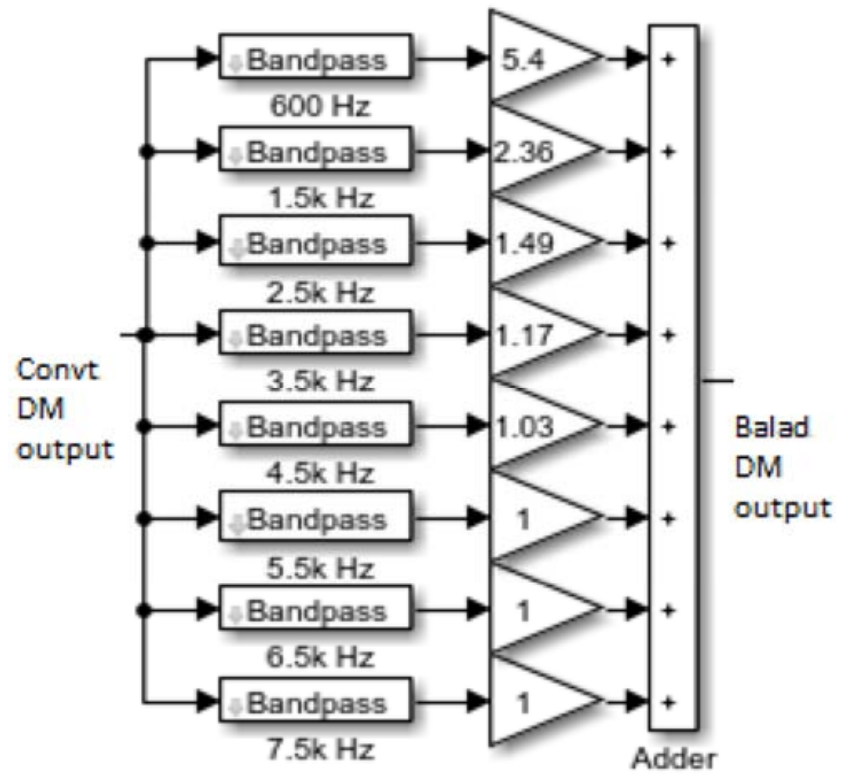

Figure 7. Multi-band multipliers for a balanced DM.

Figure 8 shows polar patterns of a balanced DM with three tones of frequencies $5 \mathrm{k}, 2 \mathrm{k}$ and $500 \mathrm{~Hz}$. The patterns have a zero notch at incident $180^{\circ}$ and nearby the same gain, $6 \mathrm{~dB}$ at $0^{\circ}$. Thus, it performs with good directivity and balanced frequency response in all the frequency regions, so the balanced DM can benefit in spatial and frequency domains.

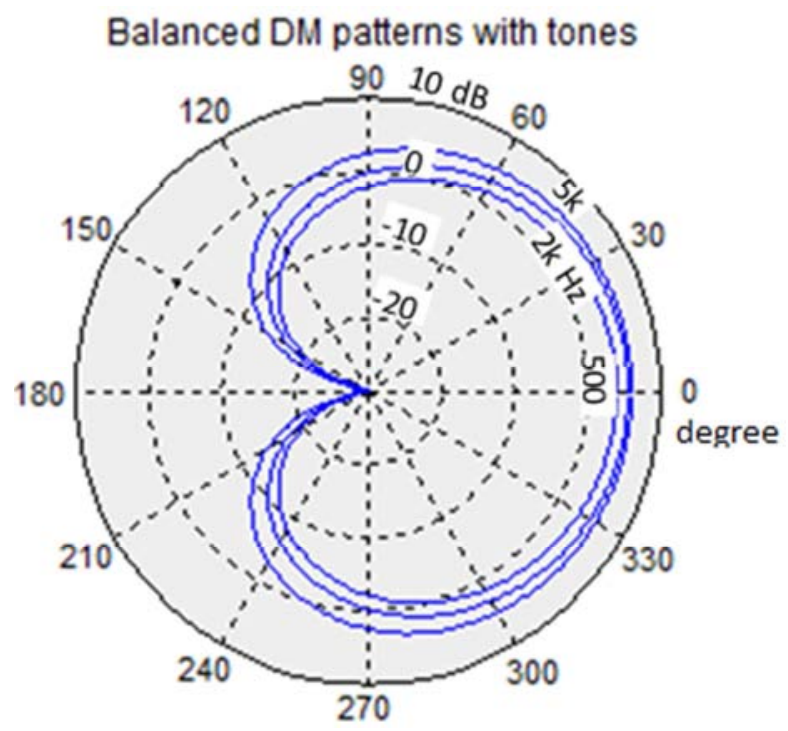

Figure 8. Polar patterns of a balanced Cardioid DM.

\section{Speech Enhancement of Common DMs}

\subsection{Analytical Study of Speech Enhancement}

DM S/N improvement can be studied on two aspects: speech enhancement and noise suppression. Here, we study the former. Using Eqs. (2), (6) and the multi-band multipliers of Figure 7, we calculated S-gains of the three DMs plus
Omni mic as a benchmark. Figure 9 shows the resulting Sgain frequency responses at incident $0^{\circ}$. The test conditions were sampling rate $44.1 \mathrm{k} \mathrm{Hz}$ and DM ports' spacing $16 \mathrm{~mm}$. From this figure, we can observe that

(1) the Omi mic has a flat curve of $0 \mathrm{~dB}$;

(2) the conventional DM has a $6 \mathrm{~dB} /$ octave up slope curve in low-mid frequency region;

(3) the 2nd-order DM has a $12 \mathrm{~dB}$ /octave up slope curve in low-mid frequency region;

(4) the balanced DM has a saw-like, flat curve around 6 $\mathrm{dB}$.

Note: the saw fluctuation is related to number, center of the frequency bands, as well as the operation word-length; hardware DM will smooth the curve well. Figure 9 also tell us shows that 1) the curves of the conventional and 2nd-order DMs cross the curve of the Omni mic at frequency $1.78 \mathrm{kHz}$; so, when speech signals go through the conventional or 2 ndorder DM, they may not be enhanced well as through the Omni mic; 2) the balanced DM performs around $6 \mathrm{~dB}$ speech enhancement; and 3) the summit frequencies of the conventional and 2nd-order DMs are the same, 5.36k Hz. The conventional DM may cause severe speech spectrum distortion [11]. From the slopes in Figure 9, the spectrum distortion of the 2nd-order DM is deteriorated, compared to the 1st-order DM, thus eliminating the need for further study on the 2nd-order DM.

Furthermore, Figure 10 shows other S-gain frequency responses of the three DMs and the Omni mic at incident $90^{\circ}$. Compared to Figure 9, we can observe how many dB the main lobe drops at $\pm 90^{\circ}$. For example, when the lobe width is $\pm 90^{\circ}$ and frequency is $2 \mathrm{k} \mathrm{Hz}$, the conventional DM gain drop is about $5.6 \mathrm{~dB}$, the balanced $\mathrm{DM}, 6 \mathrm{~dB}$, and the 2nd-order $\mathrm{DM}, 11.3 \mathrm{~dB}$. The data indicate that the DMs' spatial resolutions are not high to differentiate among sounds.

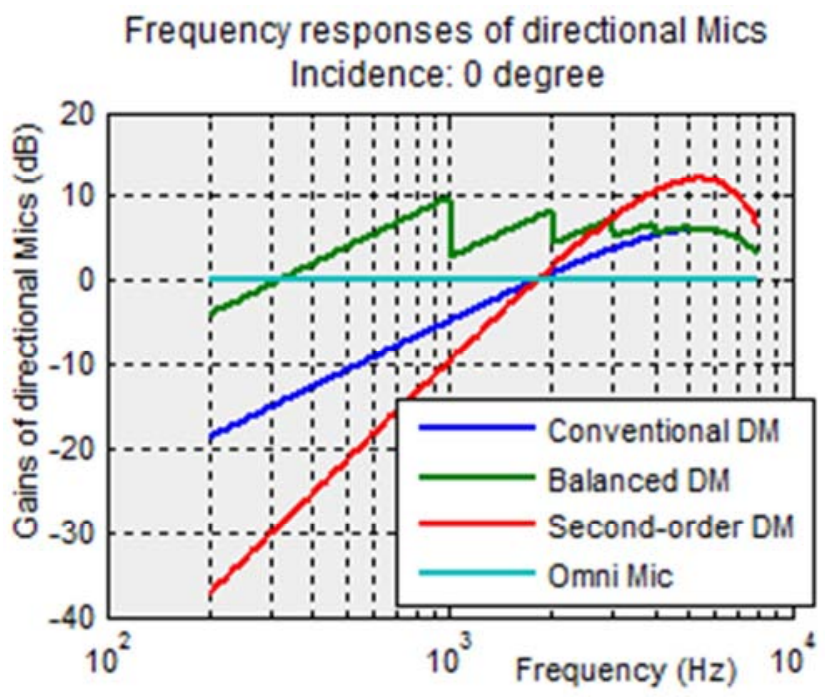

Figure 9. S-gain frequency responses of three DMs and Omni mic at $0^{\circ}$. 

Frequency responses of directional Mics
Incidence: 0 degree

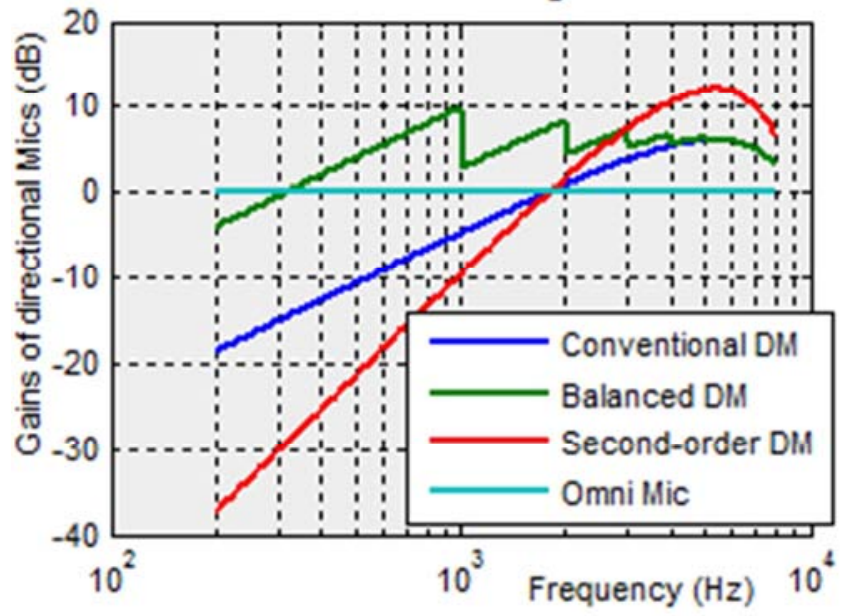

Figure 10. S-gain frequency responses of three DMs and Omni mic at $90^{\circ}$.

\subsection{Simulating Experiments for Speech Enhancement}

A pure tone is a sound signal of impulse autocorrelation and line spectrum. In Figure 9, the gain response of the conventional DM crosses the response of the Omni mic at $1.78 \mathrm{k} \mathrm{Hz}$, so we cannot indicate that the DM performs better speech enhancement. Simulating experiments with real-world speech can help to figure out it. For a high confidence level, a large-sample speech time-series is required. We acquired English speech, which is composed of 11-word phrase spoken by a female announcer Amy [15], i.e., "Hi, one of the available high quality texts to speech voices". Its wave file lasts about $3.8 \mathrm{~s}$ and contains about 167,500 samplings, sampling rate $44.1 \mathrm{k} \mathrm{Hz}$, word length 16 Bits.

Figure 11 shows the Amy original speech spectrum. If the cut-off frequency is defined as a $30 \mathrm{~dB}$ spectrum drop, the speech spectrum width is about $8 \mathrm{k} \mathrm{Hz}$. High energy of the spectrum distributes in the frequency region $<500 \mathrm{~Hz}$. The speech RMS was recorded as 0.0484 .

Figure 12 shows the Omni mic output spectrum with Amy speech. Compared to Figure 11, we can observe that this spectrum does not change significantly except in the frequency region $>8 \mathrm{k} \mathrm{Hz}$. This is because the mic IC amplifier and the pre-amplifier cut off the frequency components with $>8 \mathrm{k} \mathrm{Hz}$. The RMS of the Omni mic output was recorded as 0.0472 .

Figure 13 shows the conventional DM output spectrum with Amy speech. Compared to Figure 11, we can observe that the spectrum drops significantly at frequencies $<2 \mathrm{k} \mathrm{Hz}$, the spectrum is enhanced in frequency range $2 \mathrm{k} \sim 8 \mathrm{k} \mathrm{Hz}$, and the spectrum with frequencies $>8 \mathrm{k} \mathrm{Hz}$ disappears. The RMS of this DM output was recorded as 0.0275 , about $58 \%$ of the Omni mic output, indicating that this DM losses part of the speech.

Figure 14 shows the balanced DM output spectrum with Amy speech. Compared to Figure 13, we can observe that the spectrum energy in the low-frequency region is recovered, and this DM enhances energy in the frequency range $0 \sim 3 \mathrm{k}$
$\mathrm{Hz}$; the spectrum with frequencies $>8 \mathrm{k} \mathrm{Hz}$ also disappears. The RMS of the balanced DM output is recorded as 0.0570 , about $120 \%$ of the Omni mic output, so this DM does not losses but rather enhance the speech.

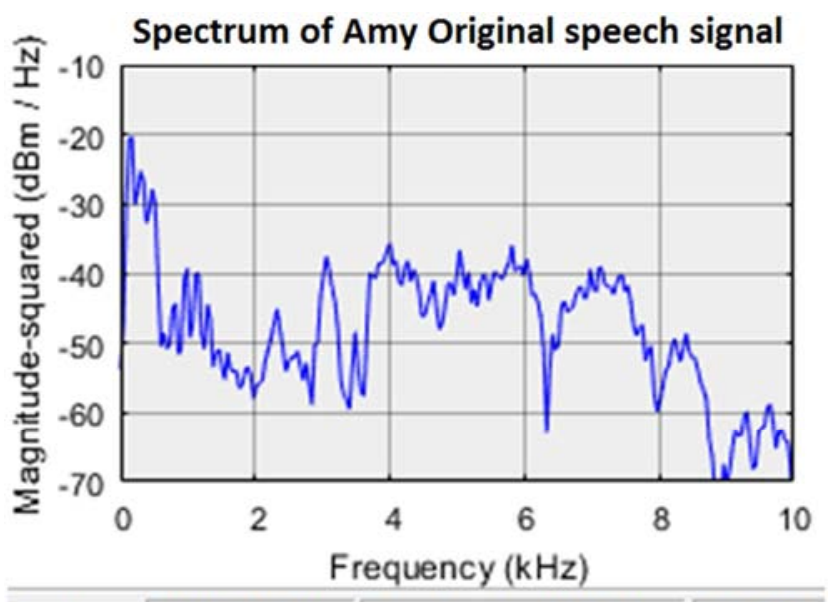

Ready RBW $=64.6 \mathrm{~Hz}$ Sample rate $=44.1 \mathrm{kHz} \quad T=3.798$

Figure 11. Spectrum of original signal of Amy speech.

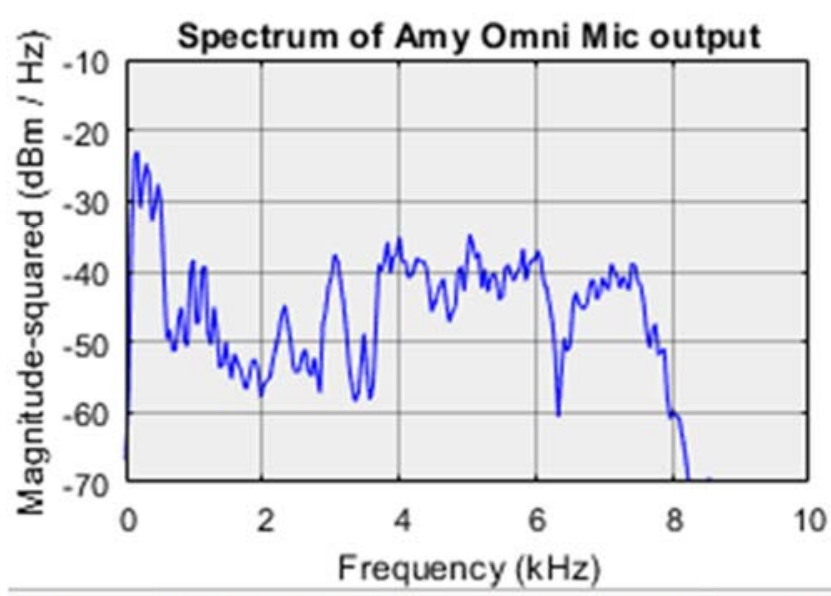

Ready RBW $=64.6 \mathrm{~Hz}$ Sample rate $=44.1 \mathrm{kHz} \quad T=3.798$

Figure 12. Spectrum of Omni Mic output with Amy speech.

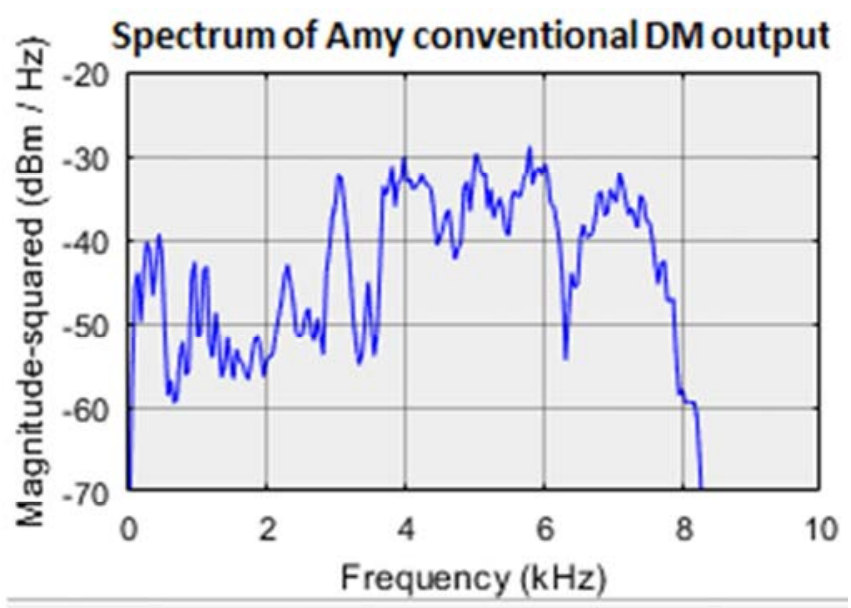

Ready RBW $=64.6 \mathrm{~Hz}$ Sample rate $=44.1 \mathrm{kHz} \quad \mathrm{T}=3.798$

Figure 13. Spectrum of conventional DM output with Amy speech. 
In a word, the conventional DM performs speech enhancement $-4.70 \mathrm{~dB}$, and the balanced $\mathrm{DM}, 1.64 \mathrm{~dB}$, relative to the Omni mic performance.

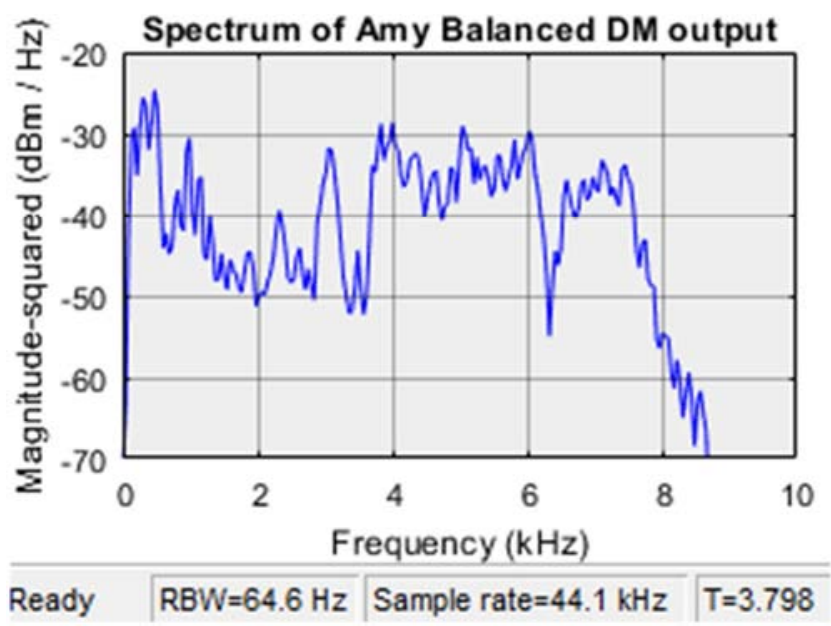

Figure 14. Spectrum of balanced DM output with Amy speech.

\section{Common DMs' Performance in Noises and Interference}

Usually, noise fields mean that there exist peripheral noises or interferences into listeners, such as party noise, equipment noise, talking interference, etc. [16]. The noises and interference probably are soft or strong in listening situations. Using hearing aids, wearers also concerns with weak internal noises of the common DMs and Omni mic. At present, the large-sample real-world noises can be easily acquired from wave files in many online references, which contain noise time-series, lasting several seconds or longer. Such noise resources can be imported to make our experiments as in real fields. We acquired voices from Amy and Brian (male announcer) in a quiet room, and wave files of both voices contain a 12-word phrase of about 176,400 samples and $4 \mathrm{~s}$ period. Considering that the two announcers' voices can represent average speech/conversation, the sound pressure of their voices should be $60 \mathrm{~dB}$ SPL. Statistics of the two acquired time-series were: Amy $\mathrm{RMS}=0.0485$, Brian $\mathrm{RMS}=0.0385$. Their mean is 0.0435 and can be used as criterion RMS equivalent to the sound pressure of $60 \mathrm{~dB}$ SPL. Logically, if RMS of another large-sample sound is equal to 0.0435 , its SPL just is $60 \mathrm{~dB}$. Thus, a noise or interference from other wave files can be calibrated conveniently with the criterion RMS.

The input configuration of a DM is showed in upper Figure 15. Because these devices are linear and timeinvariable, we can change the connection order for convenient measurement. We move the mic IC into the box Pre-amplifier. The box IC plus Pre-amplifier can be represented with a low-pass filter, as shown in lower Figure 15. The low-pass filter covers the entire speech frequency range, $0 \sim 8 \mathrm{k} \mathrm{Hz}$. Actually, its pass-band is narrow relative to the sampling rate $44.1 \mathrm{k} \mathrm{Hz}$ in our experiments, so it cuts off much of the white noise energy at mid-high frequencies.

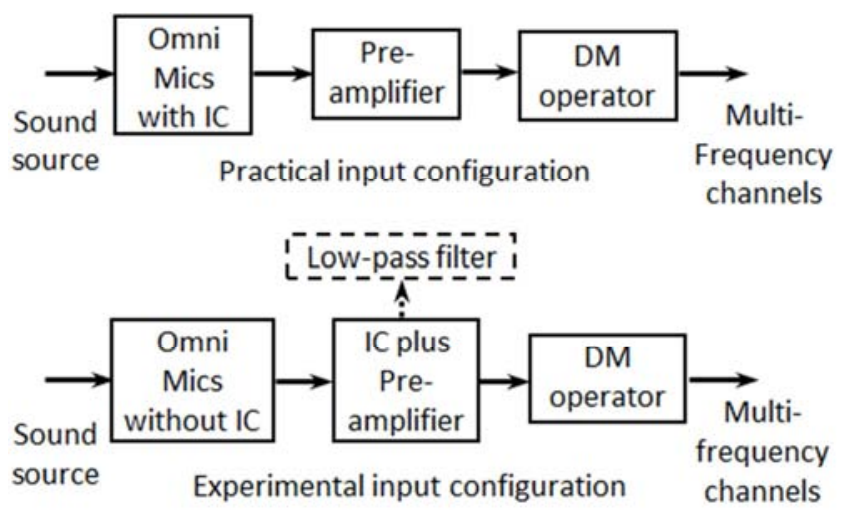

Figure 15. Configurations of DM input and its equivalence.

Based on the principles of Figure 3, Figure 7 and Figure 15, when configuring the Omni mic's and common DMs' experiments, we designed a low-pass filter and eight bandpass filters of Chebeshev II direct-type. Details on the Chebyshev Filtering block are provided in the Appendix. In Figure 16, configuration of the Omni mic experiment is shown. The input block SpchAmy60dBL.mat provided the mic output time-series. There were two recording blocks, AmySpch.mat for Amy original speech and AmyOmni.mat for the Omni mic output. In addition, two pairs of Time Scope and Spectrum Scope were used to monitor the waveforms, statistics and spectra of the input and output of the Omni mic processor, respectively.

Based on Figure 16, we inserted DM operation blocks between the two low-pass filter outputs and the multi-band processor input; the configuration of conventional DM experiment is shown in Figure 17. The upper SpchAmy60dBL.mat became the front mic output; the lower SpchAmy60dBL.mat was used for rear mic output. A spacing delay between the mic ports was connected to the rear mic output to control the back sound orientation. An extra Time Scope was connected to the input end of the internal delay for monitoring the rear mic output. Additionally, the block AmyConv.mat was used to record the conventional DM output.

Based on Figure 17, we inserted eight multipliers between the multi-band outputs and the Adder inputs; the configuration of the balanced DM experiment is shown in Figure 18. The eight gain values were 5.4, 2.36, 1.49, 1.17, 1.03 and three $1 \mathrm{~s}$ for bands $600,1.5 \mathrm{k}, \ldots, 7.5 \mathrm{k} \mathrm{Hz}$, respectively. The gain values were calculated according to the slope of the conventional DM frequency response and the balancing requirements. The block AmyBalcd.mat was used to record the balanced DM output. 


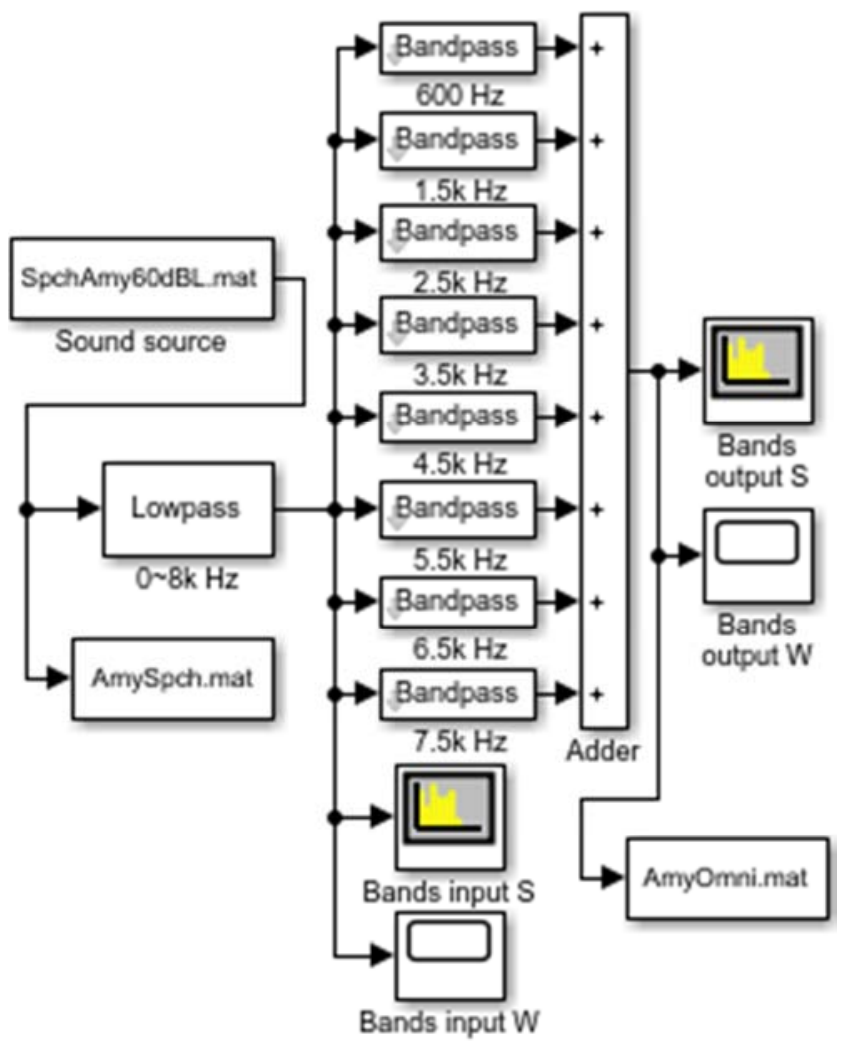

Figure 16. Configuration of an Omni mic experiment.

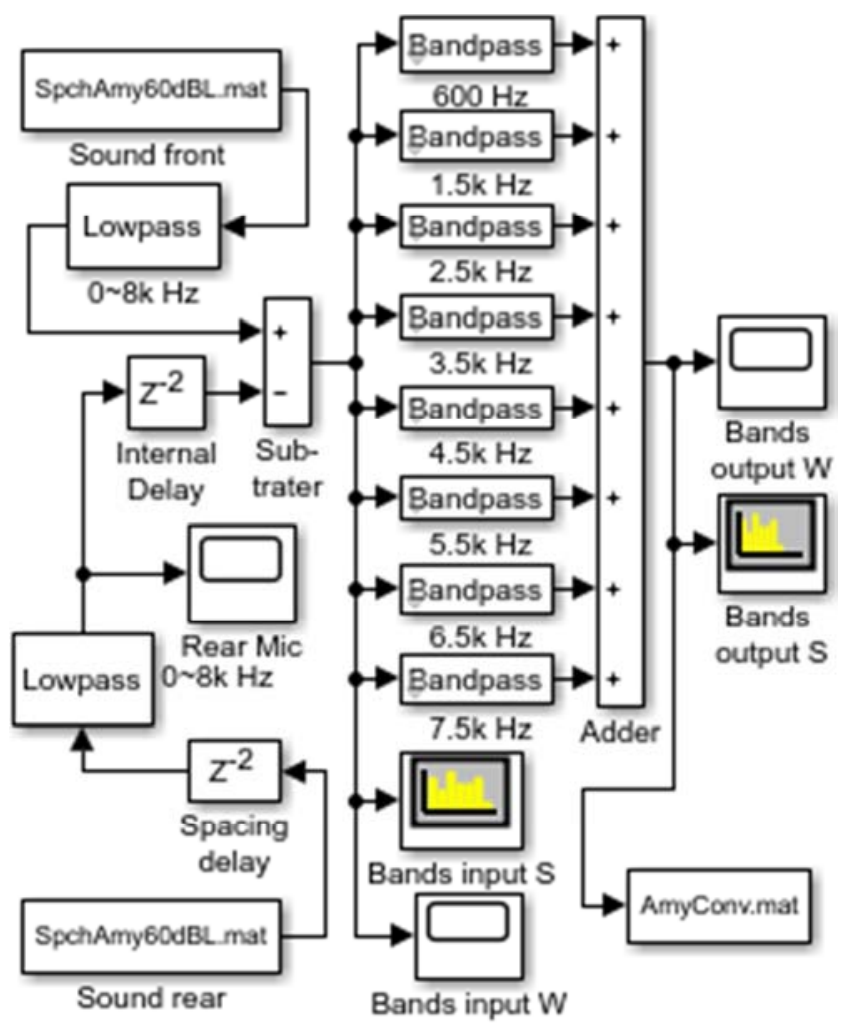

Figure 17. Configuration of a conventional DM experiment at incident $0 o$.

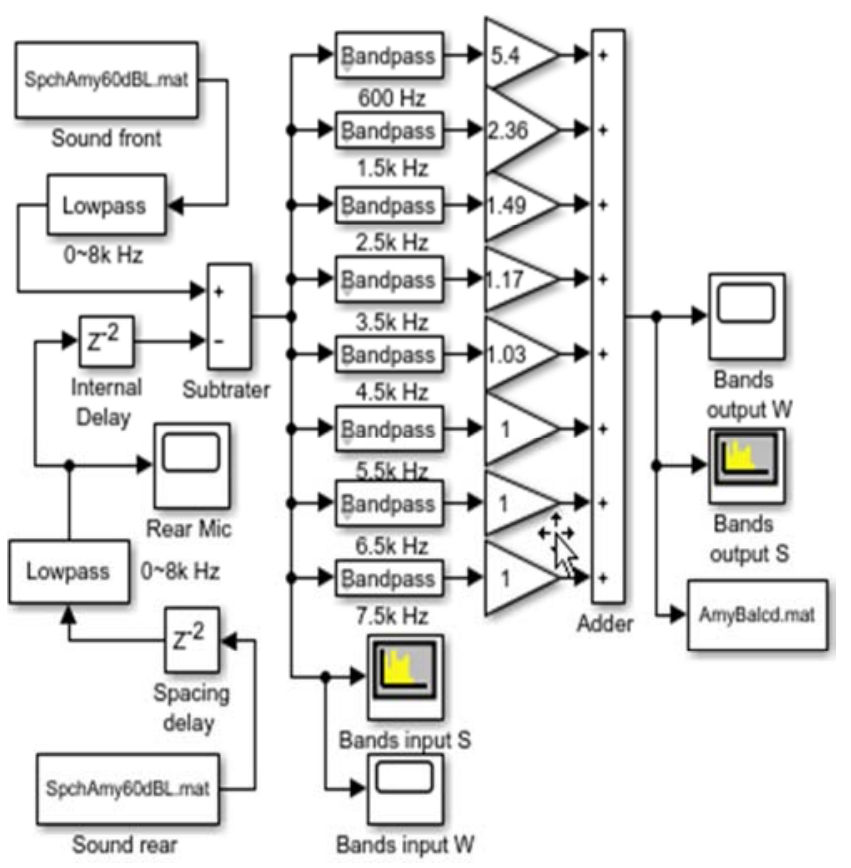

Figure 18. Configuration of a balanced DM experiment at incident $0^{\circ}$.

\subsection{DMs in a Quiet Field}

In a quiet field, the output noise of a practical hearing aid is its internal noise, related to the elements, devices and processors inside. The noise sources in the Omni mic or the DMs are critical, particularly those from mic IC, electret sensor and a pre-amplifier. Recalling Section 2, we conducted the output noise calculation for the Omni mics. In the case of the DMs, we can obtain their results through experimenting. A white noise time-series of about $4 \mathrm{~s}$ was taken from the Simulink because of verified white spectrum. It was calibrated to meet $\mathrm{RMS}=0.00138$ to ensure that the sound pressure was $30 \mathrm{~dB}$ SPL. When internal noise takes effect in the DMs, it has no orientation. We used noises at eight angles $0^{\circ}, 45^{\circ}, \ldots, 315^{\circ}$ (the more, the more exact) to represent the internal noise. Mean power of the output noises at all the angles was calculated as the end DM output. Usually, when $\mathrm{S} / \mathrm{N}$ is $9 \mathrm{~dB}$, no listening effort is needed to understand speech in noise. We specified output $\mathrm{S} / \mathrm{N}$ criterion to evaluate the mics' performance: $\mathrm{S} / \mathrm{N}<1 \mathrm{~dB}$, very poor; 1 $\mathrm{dB}<\mathrm{S} / \mathrm{N} \leq 4 \mathrm{~dB}$, poor; $4 \mathrm{~dB}<\mathrm{S} / \mathrm{N} \leq 7 \mathrm{~dB}$, fair; $7 \mathrm{~dB}<\mathrm{S} / \mathrm{N} \leq 11$ $\mathrm{dB}$, good; $11 \mathrm{~dB}<\mathrm{S} / \mathrm{N} \leq 15 \mathrm{~dB}$, very good; and $15 \mathrm{~dB}<\mathrm{S} / \mathrm{N}$, excellent. Table 1 lists the outputs and $\mathrm{S} / \mathrm{Ns}$ of the common DMs and Omni mic in the quiet field. The conventional DM output noise was 0.001 , the mid; the balanced DM, 0.00115, the highest; and the Omni mic, 0.000873, the lowest. When plus a speech, the $\mathrm{S} / \mathrm{N}$ of the conventional DM output was $27.3 \mathrm{~dB}$, the balanced DM, $33.5 \mathrm{~dB}$, and the Omni mic, 33.6 $\mathrm{dB}$; all the mics achieved excellent $\mathrm{S} / \mathrm{Ns}$. The noise drop of the common DMs resulted not only from the polar pattern suppression, but also from the low-pass filter effect. 
Table 1. Outputs and S/Ns of the DMs and Omni mic in a quiet field.

\begin{tabular}{llll}
\hline Types of sounds & Omni Mic (RMS) & Conventional DM (RMS) & Balanced DM (RMS) \\
\hline Conversation speech & 0.0418 & 0.0231 & 0.0542 \\
Internal noise & 0.000873 & 0.001 & 0.00115 \\
$\mathrm{~S} / \mathrm{N}(\mathrm{dB})$ & 33.6 & 27.3 & 33.5 \\
\hline
\end{tabular}

\subsection{DMs in Soft Noises}

When the sound pressure of a noise in a listening field is around $40 \mathrm{~dB}$ SPL, it is a soft level for average conversations. We assigned a soft babble noise and soft white noise. The former was acquired from a wave file, which was a multi-person talking record for simulating a party noise [17]; the latter was taken in the same way as in Section 5.1. The noises were calibrated to ensure that the sound pressure was $40 \mathrm{~dB}$ SPL. When an aid wearer enters a party, the noise surrounds the hearing aid from all orientations. We selected eight angles $0^{\circ}, 45^{\circ}, 90^{\circ}, \ldots, 315^{\circ}$ to represent the surrounding noise intrusion, but three of them, $225^{\circ}, 270^{\circ}$ and $315^{\circ}$, were ignored because of the shade effect of the wearer's head. Mean power of the output noises at the five angles was calculated as the end DM output. Considering that the output noises were independent time-series, their mean power was calculated based on a sum of their powers. Table 2 lists the outputs and S/Ns of the common DMs and Omni mic in the soft noises. The results indicate that 1) in the babble noise, the conventional DM output was 0.00117 , the lowest; the balanced DM, 0.00388, the mid; and the Omni mic, 0.00435 , the highest. When plus a speech, the $\mathrm{S} / \mathrm{N}$ of the conventional DM was $25.9 \mathrm{~dB}$; the balanced DM, $22.9 \mathrm{~dB}$; and the Omni mic, $19.7 \mathrm{~dB}$; all the mics achieved excellent S/Ns. 2) In white noise, the conventional DM output was 0.00317 , the mid; the balanced DM, 0.0037, the highest; and the Omni mic, 0.00276, the lowest. When plus a speech, the $\mathrm{S} / \mathrm{N}$ of the conventional DM was $17.3 \mathrm{~dB}$; the balanced DM, $23.3 \mathrm{~dB}$; and the Omni, $23.6 \mathrm{~dB}$; all the mics achieved excellent $\mathrm{S} / \mathrm{Ns}$.

Table 2. Outputs and S/Ns of the DMs and Omni mic in soft noises.

\begin{tabular}{llll}
\hline Types of sounds & Omni Mic (RMS) & Conventional DM (RMS) & Balanced DM (RMS) \\
\hline Conversation speech & 0.0418 & 0.0231 & 0.0542 \\
40dB babble noise & 0.00435 & 0.00117 & 0.00388 \\
S/N (dB) & 19.7 & 25.9 & 22.9 \\
40dB white noise & 0.00276 & 0.00317 & 0.0037 \\
S/N (dB) & 23.6 & 17.3 & 23.3 \\
\hline
\end{tabular}

\subsection{DMs in Low Noises and Low Interference}

When the sound pressure of a noise or interference in a listening field is around $50 \mathrm{~dB}$ SPL, it is a low level for average conversations. We assigned a low babble noise, a low white noise and low talking interference. The two noises were acquired in the same way as Section 5.2; the noises were calibrated to ensure that the sound pressure was $50 \mathrm{~dB}$ SPL. We used two talking interferences, which were acquired from Amy's and Brian's voices in wave files [15]. The calibration way is the same as the way in the noises. Additionally, a hearing aid can be controlled by its wearer's head to back onto the interference, so we selected the individual talking interferences at five angles $135^{\circ}, 157.5^{\circ}, \ldots, 225^{\circ}$ to represent the back intrusion, but two of them, $202.5^{\circ}$ and $225^{\circ}$, were ignored because of the shade effect of the wearer's head. Considering that the speech outputs at the three angles were correlated timeseries, their mean power is calculated based on a sum of their RMS values. We used a mean of outputs in two experiments with Amy's and Brian's voices as the end interference output. Table 3 lists the outputs and S/Ns of the common DMs and Omni mic in the low noises and low interference. The results indicated that 1) in the babble noise, the conventional DM output was 0.0037 , the lowest; the balanced DM, 0.0123, the mid; and the Omni mic, 0.0138, the highest. When plus a speech, the $\mathrm{S} / \mathrm{N}$ of the conventional $\mathrm{DM}$ was $15.9 \mathrm{~dB}$, excellent; the balanced DM, $12.9 \mathrm{~dB}$, very good; and the Omni mic, $9.6 \mathrm{~dB}$, good. 2) In the white noise, the conventional DM output was 0.01 , the mid; the balanced DM, 0.0117, the highest, and the Omni mic, 0.00873 , the lowest. When plus a speech, the $\mathrm{S} / \mathrm{N}$ of the conventional DM was $7.3 \mathrm{~dB}$, good; the balanced DM, 13.3 $\mathrm{dB}$, very good; the Omni mic, $13.6 \mathrm{~dB}$, very good. 3) In the talking interference, the conventional DM output was 0.00126 , the lowest; the balanced DM, 0.00234, the mid; the Omni, 0.0132 , the highest. When plus a speech, the $\mathrm{S} / \mathrm{N}$ of the conventional DM was $25.3 \mathrm{~dB}$, excellent; the balanced DM, $27.3 \mathrm{~dB}$, excellent; but the Omni mic, $10 \mathrm{~dB}$, good.

Table 3. Outputs and $S / N$ s of the DMs and Omni mic in low noises and interference.

\begin{tabular}{llll}
\hline Types of sounds & Omni Mic (RMS) & Conventional DM (RMS) & Balanced DM (RMS) \\
\hline Conversation speech & 0.0418 & 0.0231 & 0.0542 \\
$50 \mathrm{~dB}$ babble noise & 0.0138 & 0.0037 & 0.0123 \\
$\mathrm{~S} / \mathrm{N}(\mathrm{dB})$ & 9.6 & 15.9 & 12.9 \\
$50 \mathrm{~dB}$ white noise & 0.00873 & 0.01 & 0.0117 \\
$\mathrm{~S} / \mathrm{N}(\mathrm{dB})$ & 13.6 & 7.3 & 13.3 \\
$50 \mathrm{~dB}$ talking interference & 0.0132 & 0.00126 & 0.00234 \\
S/N $(\mathrm{dB})$ & 10 & 25.3 & 27.3 \\
\hline
\end{tabular}




\subsection{DMs in Competing Noises and Competing Interference}

When the sound pressure of a noise or interference in a listening field is around $60 \mathrm{~dB}$ SPL, it is a competing level for average conversations. We assigned a competing babble noise, a competing white noise and competing talking interference. The noises and talking interferences were acquired as previously described, and were calibrated to ensure that the sound pressure was $60 \mathrm{~dB}$ SPL. Table 4 lists the outputs and $\mathrm{S} / \mathrm{Ns}$ of the common DMs and Omni mic in the competing noises and competing interference. The results indicate that 1) in the babble noise, the conventional DM output was 0.0117 , the lowest; the balanced DM, 0.0388 , the mid; and the Omni, 0.0435 , the highest. When plus a speech, the $\mathrm{S} / \mathrm{N}$ of the conventional $\mathrm{DM}$ was $5.9 \mathrm{~dB}$, fair; the balanced DM, $2.9 \mathrm{~dB}$, poor; and the Omni mic, $-0.35 \mathrm{~dB}$, very poor. 2) In the white noise, the conventional DM output was 0.0317 , the mid; the balanced DM, 0.037, the highest; and the Omni mic, 0.0276, the lowest. When plus a speech, the $\mathrm{S} / \mathrm{N}$ of the conventional DM was $-2.75 \mathrm{~dB}$, very poor; the balanced DM, $3.32 \mathrm{~dB}$, poor; and the Omni mic, $3.6 \mathrm{~dB}$, poor. 3) In the talking interference, the conventional DM output was 0.00397, the lowest; the balanced DM, 0.0074, the mid; and the Omni mic, 0.0418 , the highest. When plus a speech, the $\mathrm{S} / \mathrm{N}$ of the conventional DMs was $15.3 \mathrm{~dB}$, excellent; the balanced DM, $17.3 \mathrm{~dB}$, excellent; but the Omni mic, $0 \mathrm{~dB}$, very poor.

Table 4. Outputs and S/Ns of the DMs and Omni mic in competing noises and interference.

\begin{tabular}{llll}
\hline Types of sounds & Omni Mic (RMS) & Conventional DM (RMS) & Balanced DM (RMS) \\
\hline Conversation speech & 0.0418 & 0.0231 & 0.0542 \\
60dB babble noise & 0.0435 & 0.0117 & 0.0388 \\
S/N (dB) & -0.35 & 5.9 & 2.9 \\
60dB white noise & 0.0276 & 0.0317 & 0.037 \\
S/N (dB) & 3.6 & -2.75 & 3.32 \\
60dB talking interference & 0.0418 & 0.00397 & 0.0074 \\
S/N (dB) & 0 & 15.3 & 17.3 \\
\hline
\end{tabular}

\subsection{DMs in Strong Noises and Strong Interference}

When the sound pressure of a noise or interference in a listening field is around $70 \mathrm{~dB}$ SPL, it is a strong level for average conversation. We assigned a strong babble noise, a strong white noise and strong talking interference. The noises were at 5 incident angles and the interferences were at 3 incident angles, as described in Section 5.4. The noises and the interference were calibrated to ensure that the sound pressure was $70 \mathrm{~dB}$ SPL. Table 5 lists the outputs and S/Ns of the common DMs and Omni mic in the strong noises and strong interference. The results indicate that 1 ) in the babble noise, the conventional DM output was 0.037 , the lowest; the balanced DM, 0.123, the mid; and the Omni mic, 0.138, the highest. When plus a speech, the $\mathrm{S} / \mathrm{N}$ of the conventional $\mathrm{DM}$ was $-4.1 \mathrm{~dB}$; the balanced DM, $-7.1 \mathrm{~dB}$; the Omni mic, -10.4 $\mathrm{dB}$; and all the mics achieved very poor $\mathrm{S} / \mathrm{N}$. 2) In the white noise, the conventional DM output was 0.101, the mid; the balanced DM, 0.117, the highest; and the Omni mic, 0.0873, the lowest. When plus a speech, the $\mathrm{S} / \mathrm{N}$ of the conventional DM was $-12.8 \mathrm{~dB}$, the balanced DM, $-6.7 \mathrm{~dB}$; the Omni mic, $-6.4 \mathrm{~dB}$; and all the mics achieved very poor $\mathrm{S} / \mathrm{N}$. 3) In the talking interference, the conventional DM output was 0.0126 , the lowest; the balanced DM, 0.0234, the mid; and the Omni mic, 0.132 , the highest. When plus a speech, the $\mathrm{S} / \mathrm{N}$ of the conventional DM was $5.26 \mathrm{~dB}$, fair; the balanced DM, 7.3 $\mathrm{dB}$, good; but the Omni mic, $-10 \mathrm{~dB}$, very poor.

Table 5. Outputs and S/Ns of the DMs and Omni mic in strong noises and interference.

\begin{tabular}{llll}
\hline Types of sounds & Omni Mic (RMS) & Conventional DM (RMS) & Balanced DM (RMS) \\
\hline Conversation speech & 0.0418 & 0.0231 & 0.0542 \\
$70 \mathrm{~dB}$ babble noise & 0.138 & 0.037 & 0.123 \\
S/N (dB) & -10.4 & -4.1 & -7.1 \\
$70 \mathrm{~dB}$ white noise & 0.0873 & 0.101 & 0.117 \\
S/N (dB) & -6.4 & -12.8 & -6.7 \\
$70 \mathrm{~dB}$ talking interference & 0.132 & 0.0126 & 0.0234 \\
S/N (dB) & -10 & 5.26 & 7.3 \\
\hline
\end{tabular}

\section{Distortion of Common DMs}

Significant spectrum distortion of a conventional DM was illustrated with two-word English phrases [11]. Here we selected the large-sample, real-world speech of $3.8 \mathrm{~s}$, as described in section 4.2. In order to compare waveforms and spectra of the original speech to those of the Omni mic, the conventional DM and balanced DM outputs, we recorded data at four test points in Figure 16 to Figure 18. No.1 was the original speech output, recorded into block AmySpch.mat. No.2 was the Omni mic output, recorded into block AmyOmni.mat. The both were done in the Omni mic experiment of Figure 16. No.3 was the conventional DM output, recorded into block AmyConv.mat placed at the Adder output in Figure 17. No.4 was the balanced DM output, recorded into block AmyBalcd.mat placed at the Adder output in Figure 18. The four blocks were To File type in Simulink, and were saved in Matlab Workplace after running. They needed to be written into wave files for future viewing and listening. For details of the wave file creation, refer to the Appendix. 


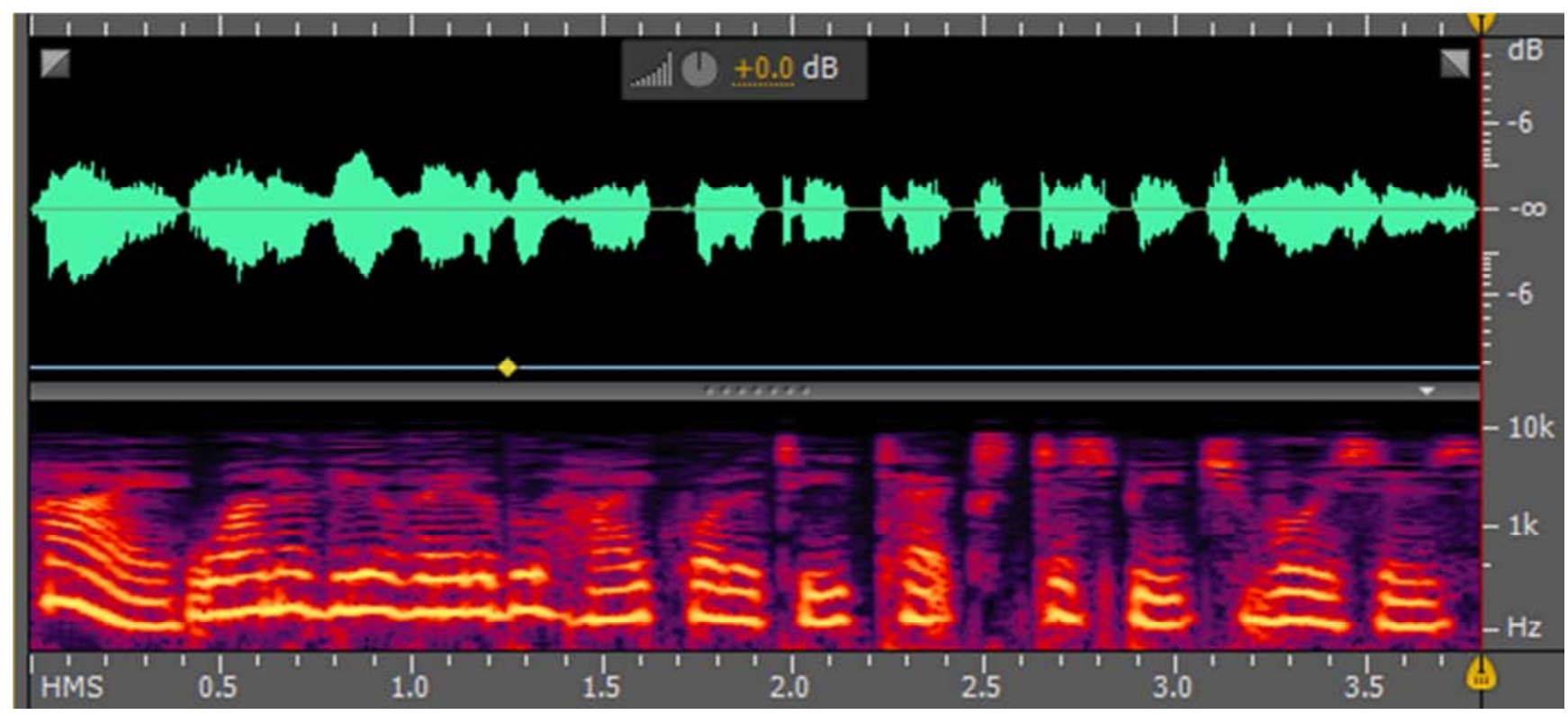

Figure 19. Waveform and spectrum of the original signal of Amy speech.

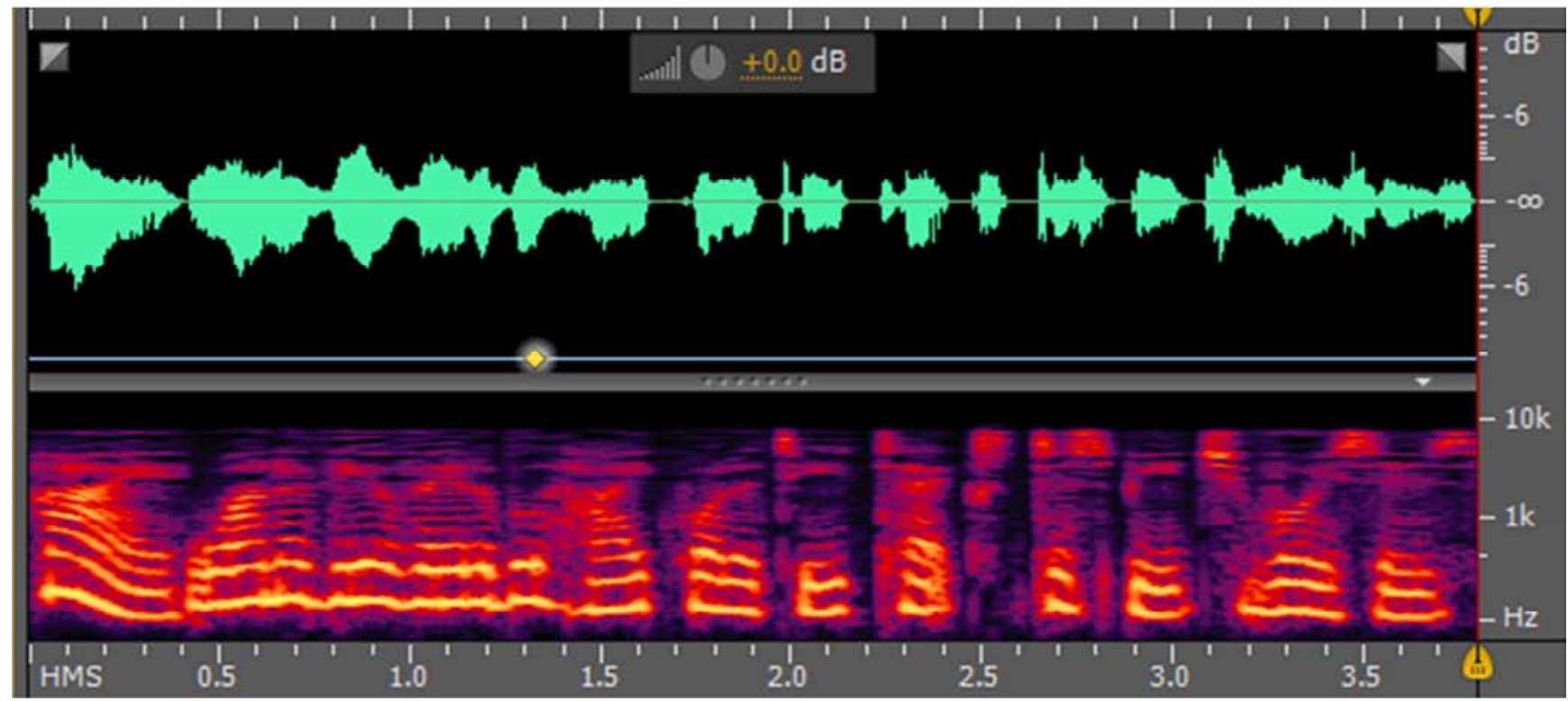

Figure 20. Waveform and spectrum of the Omni mic output with Amy speech.

It is convenient to test the waveforms and spectra by using the Adobe SoundBooth. Figure 19 shows the waveform (upper) of the original speech of Amy, which was the criterion waveform for our evaluations. The gaps between the two-word waveforms are nothing, and the envelope of the speech waveform are deep, indicating clean speech; the spectrum(lower) shows that high energy is in the low-mid frequency region. Figure 20 shows the waveform and spectrum of the Omni mic output. We observe that the waveform and spectrum have little distortion, compared to the criterion waveform. Thus, the Omni mic preserves the input speech fidelity very well. Figure 21 shows the waveform and spectrum of the conventional DM output. The waveform has significantly distorted: some word waveforms are declined, while the others are expanded, depending on frequency components of the word waveforms [11]. Figure 22 shows the waveform and spectrum of the balanced DM output. The entire waveform is enhanced by $\max 6 \mathrm{~dB}$, compared to the criterion waveform, and the magnified waveform of Figure 22 preserves the fidelity of the original speech. Furthermore, we also listened to the speeches during all the playbacks, and could not perceive distortion except the speech in Figure 21, which sounded much different from the original speech in Figure 19: the high pitches were significantly increased. These findings are consistent with the frequency responses of the common DMs. 


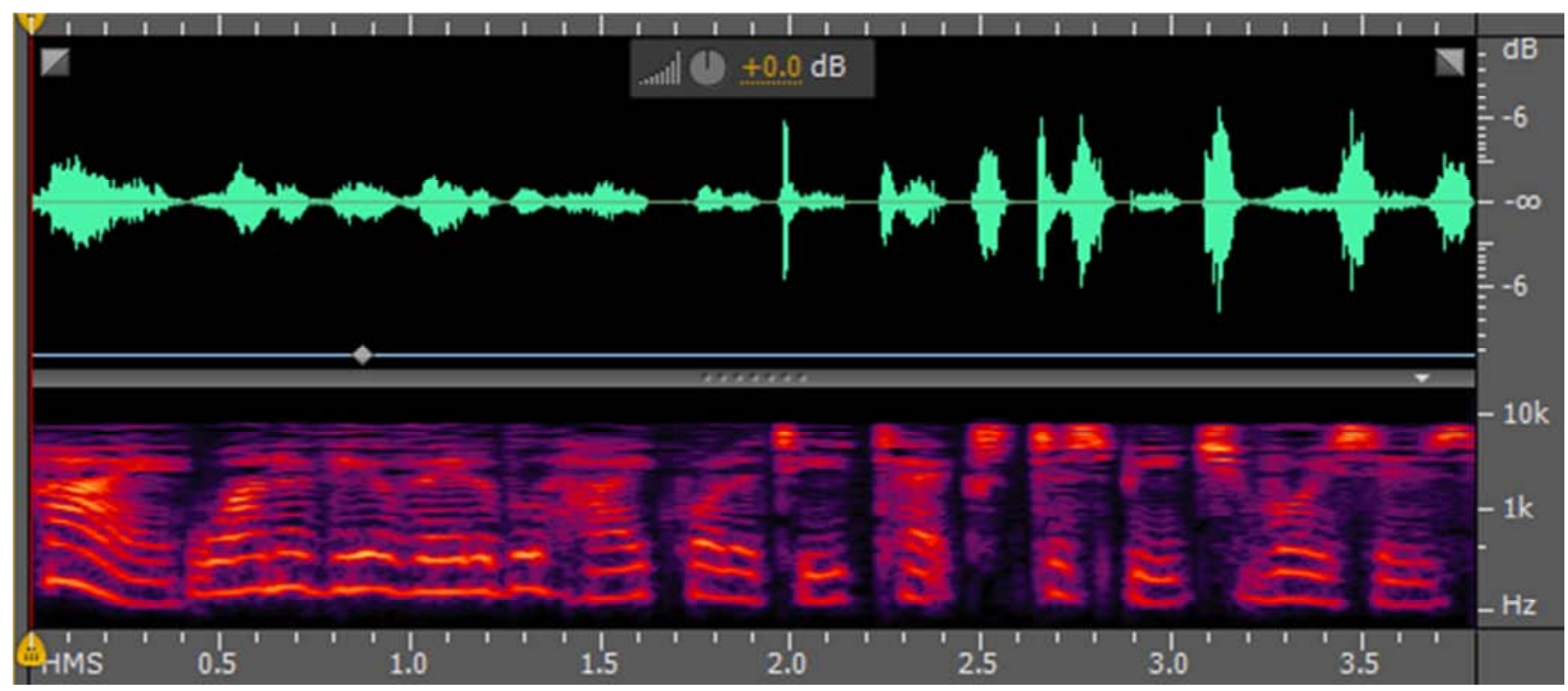

Figure 21. Waveform and spectrum of the conventional DM output with Amy speech.

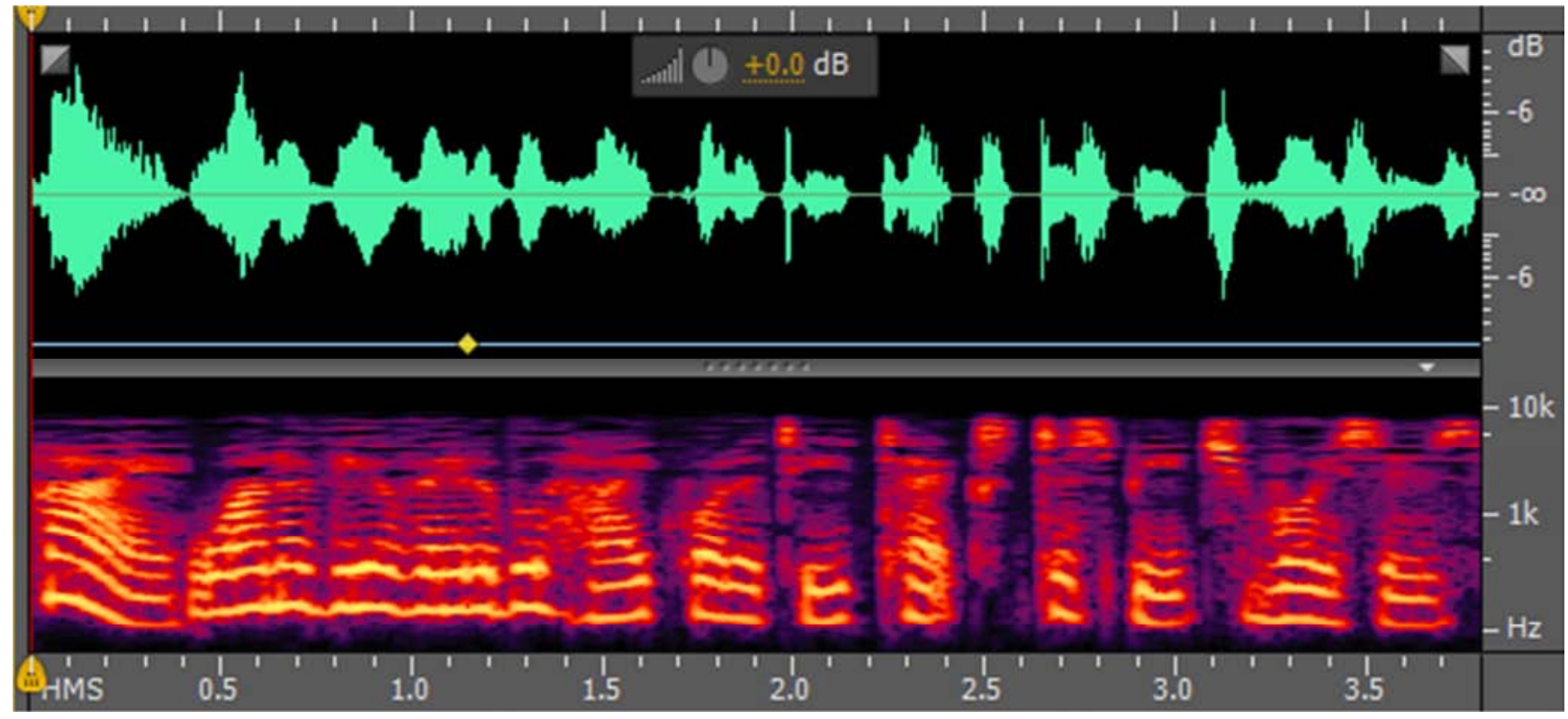

Figure 22. Waveform and spectrum of the balanced DM output with Amy speech.

\section{Conclusions}

The data, waveforms, spectra and graphs acquired through our experiments facilitated our evaluation of the benefits and limitations of the common DMs.

(1) The conventional DM does not perform speech enhancement. The large-sample experiments show that its speech output is $-4.7 \mathrm{~dB}$ relative to the Omni mic output. The balanced DM does perform large speech enhancement, $1.64 \mathrm{~dB}$ relative to the Omni mic.

(2) For a conversation in the quiet field, in the soft or low noise, the common DMs can suppress the intruding noises, and the effectiveness is limited, similar to that of the Omni mic. The common DMs and Omni mic all achieve every good or good $\mathrm{S} / \mathrm{N}$ for understanding speech. Thus, aid wearers may prefer the Omni mode or DM modes because of no significant difference.

(3) For a conversation in the low, competing or strong talking interference, the common DMs suppress the intruding interference very well, and their output noises are much lower than the Omni mic noise. The DMs achieve excellent or very good or good $\mathrm{S} / \mathrm{Ns}$, about $16 \mathrm{~dB}$ higher than the Omni mic does. In the talking interference, aid wearers prefer the DM mode to the Omni mode.

(4) For a conversation in the competing or strong noise, the common DMs suppress surrounding noises to a very limited degree, and their output noises are around Omni mic noise. The DMs and Omni mic sparsely achieve fair or poor or very poor S/Ns, 6 to $-13 \mathrm{~dB}$. Thus, neither the DMs nor Omni can help with better speech intelligibility in a party noise, big fan noise or reverberant noise surrounding. Aid wearers would not prefer the DM nor 
Omni mode.

(5) In various sound fields, the Omni mic preserves the speech fidelity very well, the balanced DM does well, and the conventional DM does poorly. Thus, aid wearers prefer the Omni mode to the DM modes if the output $\mathrm{S} / \mathrm{Ns}$ are close, and they prefer the balanced DM to conventional DM.

The common DMs are a type filter in the spatial domain; the lobes of the DMs' polar patterns are wide, about $\pm 80^{\circ}$ with $3 \mathrm{~dB}$ gain drop. Effectiveness to suppress a surrounding noise, e.g., party noise, improves a little on the Omni mic. However, when an interference is intruding as a beamed sound, e.g., individual talking interference, the DMs suppress it effectively, even at a strong level.

\section{Appendix}

Digital filter designs and wave file creation for DM experiments

An approach to use Simulink(2012a) blocks to configure DM experiments was described, including researches on gain polar plots, spectrum distortions, etc., with real-world sounds [11]. Based on upgraded requirements in this paper, we configured new experiments, such as large-sample statistic calculation of 4 s period, about 176,400 samplings, digital filters design, spectrum analysis, stereo wave files creation, etc., so we selected Matlab 2017b for our Simulink Lab ${ }^{1}$, the previous version 2012a is not available at present.

\section{Appendi 1. Designs of Low-Pass and Multi-Band Filters}

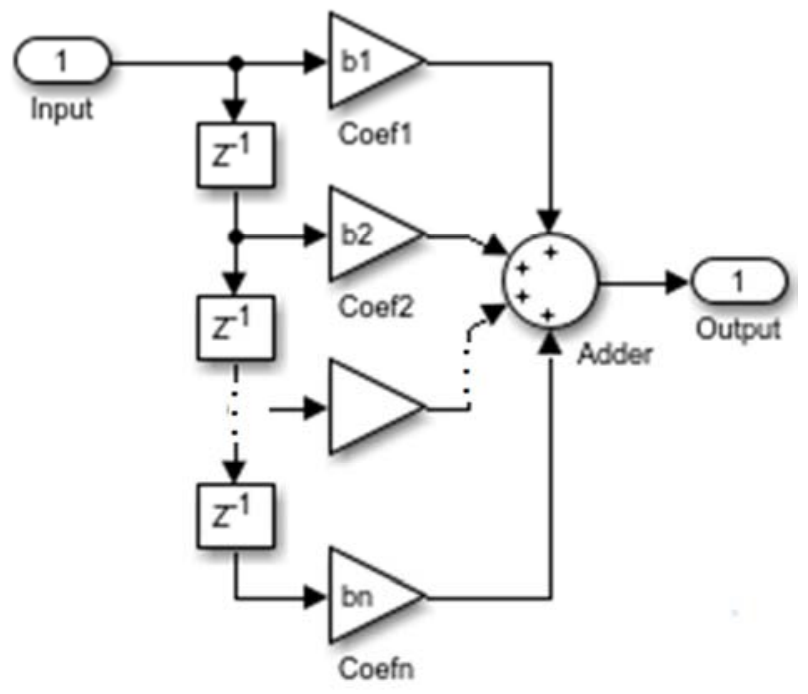

Figure A1. Structure of a FIR direct-form filter.

A digital filter can be designed to have different functions, such as band-pass, low-pass or high-pass, etc. In input processing of the DMs and Omni mic, a low-pass filter was required to get $\mathrm{S} / \mathrm{N}$ benefit, as described in the text. We always selected FIR (finite impulse response) Direct-form, the simplest

\footnotetext{
${ }^{1}$ SimuLink Lab is a nonprofit laboratory with PC computers, various DSP software, etc.
}

structure, as shown in Figure A1. SimuLink 2017b provides a Low-pass Filtering block. Before running it, we needed to set up its parameters: Type, Chebeshev II; Pass-band gain, $0 \mathrm{~dB}$; Ripples, $0.1 \mathrm{~dB}$; Pass-band edge, $8 k \mathrm{~Hz}$; Stop-band edge, $10 \mathrm{k} \mathrm{Hz}$ and Stop-band attenuation, $-40 d B$. By clicking a left box Review Response, we verified some characteristics of the resulting filter and what we concerned about, e.g., the delay time is 20 samples, $0.453 \mathrm{~ms}$.

Multi-band filters for a balanced DM are composed of many band-pass filters, which can be FIR filter bank or FFT structure; in fact, each channel of FFT also is a FIR filter. We did not use IIR(infinite impulse response) and octave filtering structure because of long group delays. The long and stagger delays may cause severe waveform distortion when multiband summing. For our experiments, eight band-pass filters were designed, their center frequencies are $600,1.5 \mathrm{k}, \ldots, 7.5 \mathrm{k}$ $\mathrm{Hz}$, and their bandwidths are equal, $1 \mathrm{k} \mathrm{Hz}$ except that the $1 \mathrm{st}$ one has a width $800 \mathrm{~Hz}$. The 8 filters cover a frequency range of $200 \sim 8 \mathrm{k} \mathrm{Hz}$, which is enough wide to pass almost all components of speech spectra [18]. When setting up the band-pass blocks in Figure 16 and Figure 17 and Figure 18, we needed to specify many specifications and to select options: Order mode, Minimum; Filter type, Single-rate; Frequency 4 parameters: Fpass1, $2 k H z$, Fpass2, $3 k H z$ (for the 3rd filter), Fpass1-Fstop1= $250 \mathrm{~Hz}$, Fstop2-Fpass2=250 $\mathrm{Hz}$ (for all the filters); Frequency units, Absolute frequency $H z$; Magnitude units, $d B$; Input processing, Elements as channels; Data type, Input Double-precision floating point, Output Double-precision floating point. In order to minimize their delay time, we selected: Type, Equal ripple Chebyshev $I I$; Stop-Band attenuation, $25 d B$; and the Pass-Band Ripple, $1 \mathrm{~dB}$. After clicking a box Apply, the designed filter will be running; then clicking a box Review Response, we can view various characteristics of this band-pass filter. Eight filters were set up separately. Figure A2 shows one of the designed band-pass filters' responses, which has a center frequency $2.5 \mathrm{k} \mathrm{Hz}$. We also can view Delay Time and Order number, etc., by clicking the specification in menu.

When summing outputs of the filters, interactions of output phases may cause big difference between the design ripples and the tested ripples. Thus, integrated frequency response of the multi-band filters must be verified before applying them. Fpass1 and Fpass2 of each filter need to be adjusted until the ripples of the integrated response meet \pm 1.2 $\mathrm{dB}$. The resulting delay was about 80 samples, $1.8 \mathrm{~ms}$. When we selected the octave filter or IIR filter blocks, the delay time was about 200 samples.

\section{Appendi 2. Manipulations of Reading and Writing Wave Files}

When starting an experiment of Figure 16, Figure 17 or Figure 18, the input blocks of From-File in Workplace must be invoked as sound sources. In order to prepare the sources, we needed some readable time-series files of speech voices, field noises and device noise, etc. Here we give an example to illustrate. In Matlab 2017b, some syntaxes to manipulate wave 
files have been updated from the previous versions. Assign Amy wave file name to be Amy60dB4s.wav, the following syntaxes are available.

$b y=$ audioread('Amy60dB4s.wav');

$t=0.000022676^{*}(1: 176400)$;

$b y=b y^{\prime}$;

$a=b y(1: 352800)$;

for $n=1: 176400$

$a 1(n)=a(2 * n-1)$;

$a 2(n)=a(2 * n)$;

end

bana $=t ; a 1]$;

banb $=t ; a 2]$,

AmyL =timeseries $(\operatorname{bana}(2,:)$, bana $(1,:))$;

AmyR=timeseries $(\operatorname{banb}(2,:), \operatorname{banb}(1,:))$;

Because the Amy wave file was recorded in stereo channels, we had to read data from both left and right channels into a column vector $b y$, and to split into two vectors $A m y L$ and $A m y R$. Then, we needed to build a simple model in SimuLink, which was composed of only two directly-connecting blocks. A block of From Workplace, AmyL, read the vector AmyL; another block of To File, SpchAmy.mat, recorded the data from the block AmyL when the model was running.

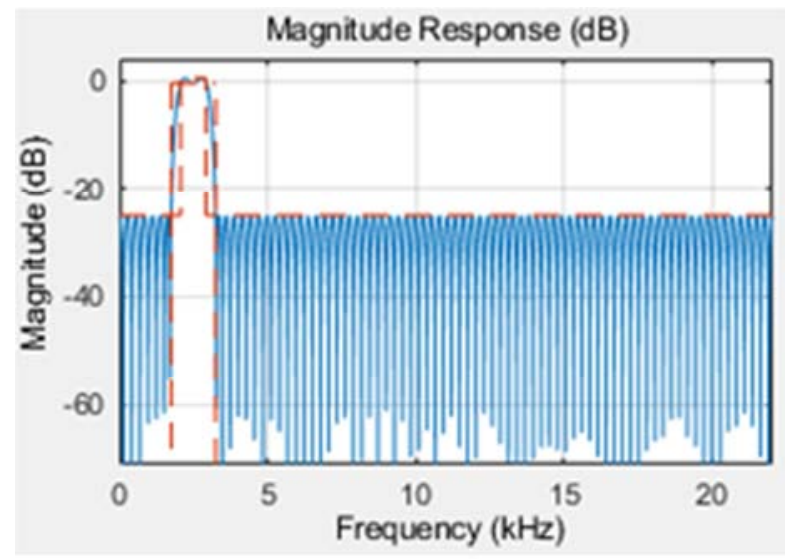

Figure A2. Magnitude response of a band-pass filter with center $2.5 \mathrm{k} \mathrm{Hz}$

In each of our experiments, there were two pairs of Time Scope and Spectrum Scope to be connected to the input and output ends of the multi-band processing. So, it was easy to view behaviors of the Omni mic and the DMs when running. For a backup of the experimental results, we needed to record these data into wave files. This required a reverse manipulation to the above syntaxes. For example, when the experiment of Figure 16 was done, all the Omni mic output data had been recorded into the block AmyOmni.mat. The following is syntaxes which write a wave file with the block mat-file. load AmyOmni.mat

y3(1,:)=AmyLPOmni.data;

$y b=y 3(1,:)$;

soundsc $(y b, 44100)$

pause

audiowrite('AmyLOmni.wav', yb,44100)

where load means to open the mat-file AmyOmni.mat in Workplace. The syntax $y 3(1,:)=$ AmyLPOmni.data; means to take only data from time-series AmyLPOmni; the name AmyLPOmni was assigned when we set up the parameters of the block AmyOmni.mat. The syntax audiowrite ('AmyLOmni.wav', yb,44100) means to use data of vector $y b$ and sampling rate $44.1 \mathrm{k} \mathrm{Hz}$ to write a wave file, whose name is AmyLOmni.wav. soundsc $(y b, 44100)$ and pause are to listen to the sound before writing the wave file. In the same way, we can write the other three wave files with the mat-files AmyConv.mat, AmyBalcd.mat and AmySpch.mat, which were acquired in the experiments of Figure 17, Figure18 and Figure16, respectively. Adobe SoundBooth CS4 is a type excellent audio analyzer and audio signal editor, and imports sounds by reading wave files. For details of listening to sounds, viewing waveforms and analyzing spectra, refer to the SoundBooth Helps.

\section{Appendi 3. Wave File Creation and Playback of Double Channels}

After opening a wave file, its waveform appears in a track. In order to compare another waveform, we can select Add an audio Track from a small menu in the upper left of the Editor panel, then we open a 2nd wave file and its waveform appears in another track below. If there exists a big difference between two waveforms, we can easily observe it. For example, the waveforms in Figure 21 and Figure 19, some word waveforms are expanded significantly, and some word waveforms are declined significantly. However, if differences between two waveforms are very tiny, it is difficult to recognize where the differences are. For example, the Amy original speech in Figure 19 and the Omni mic output in Figure 20 are almost the same; then, we cannot recognize either of the waveforms by viewing separately. Instead of the MultiTracks playing, we can write two waveforms into two channels of "stereo" sounds, respectively, then play and view them in the same track. The following syntaxes write the Amy original speech into left channel and write the Omni mic output into right channel.

load AmySpch.mat

y3(1,:) = AmySound.data;

$y b=y 3(1,:)$;

$\operatorname{soundsc}(y b, 44100)$

pause 
load AmyOmni.mat

y3(2,:)=AmyOmni.data;

$y b=y 3(2,:)$;

soundsc $(y b, 44100)$

pause

$y 4=y 3^{\prime} ;$

audiowrite('AmyLSD\&Omni3_8s.wav', y4,44100)

where the $y 4$ is a two-column vector; $y 3(1,:)$ contains Amy original speech, and $y 3(2,:)$ contains the Omni mic output. Such resulting wave file AmyLSD\&Omni3 8s.wav contains "stereo" sounds, as shown in Figure A3, and can be viewed and differentiated. Before playing the stereo sound, we selected View/Channel/Layer on the main menu of SoundBooth CS4. We can observe that the two waveforms are overlapping together, but their spectra are separated; the green waveform is the original speech, the blue one is the Omni output, and the dodger blue one is the overlapping area. As a result, the differences between the two waveforms appear clearly, the both are very close(the dodger blue is almost $100 \%$ area) but not the same. Using such stereo creation, we also can easily recognize artifacts caused by a DSP processor.

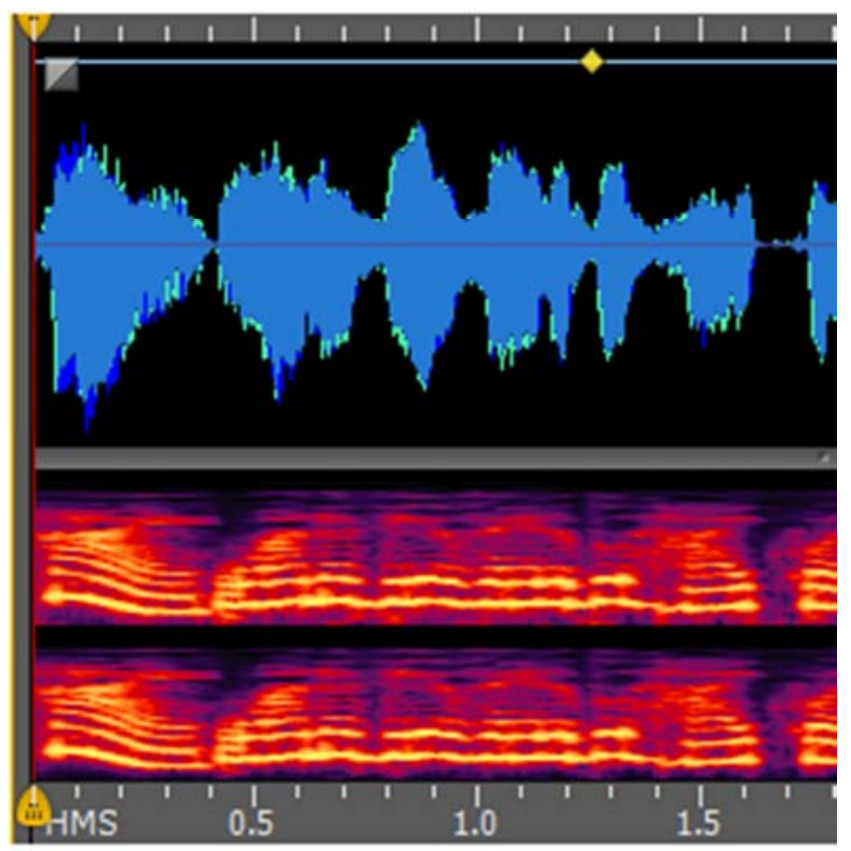

Figure A3. Layered waveforms and spectra of Amy speech and the Omni mic output.

\section{References}

[1] $\mathrm{Wu}, \mathrm{Y} . \mathrm{H}$. Gaining new insights about directional hearing aid performance, 20Q with Gus Mueller, 2013, Feb. www.audiologyonline.com.

[2] Chalupper, J., Wu, Y. H. Improving effectiveness of directional microphones with soft level directivity, Hear J., 2011, 64(7), 4449. Doi:10.1097/01.hj.0000399880.84848.a1.

[3] Nyffeler, M., Dechant, S. Field study on user control of directional focus: benefits of hearing the facets of a full life, Hear Rev., 2008, 16(1), 24-28.

[4] Nyffeler, M. Auto ZoomControl - Automatic change of focus to speech signals of interest, Field Study News, Sept. 2010. www.phonakpro.com.

[5] Jorge Mejia, Harvey Dillon, Elizabeth Beach, Margo McLelland, Mridula Sharma, et al. Loss of speech perception in noise -- causes and compensation, International symposium on auditory and audiological research (ISAAR) 2015, 205-215.

[6] Lindley, G., Schum, J. D., Fuglholt, L. M. Directionality and noise reduction in pediatric fittings, Hearing Review, 2009, 16(5), 34-44.

[7] Chalupper, J., Wu, Y. H., Weber, J. New algorithm automatically adjusts directional system for special situations, The Hearing Journal, 2011, 64(1), 26-33. Doi:10.1097/01.hj.0000393211. 70569.5c.

[8] Xubao Zhang, Yu-Hsiang Wu. Technologies and performances of directional microphones in modern hearing aids, Journal of Audiology and Speech Pathology, 2015, 23(3), 301-306.

[9] Gnewikow, D., Ricketts, T., Bratt, W. G., Mutchler, C. L. Realworld benefit from directional microphone hearing aids, J. Rehabil Res Dev., 2009, 46(5), 603- 618. Doi:10.1682/jrrd.2007.03.0052.

[10] Bentler, A. R. Effectiveness of directional microphones and noise reduction schemes in hearing aids: a systematic review of the evidence, J. Am Audiol., 2005, 16, 473-484. Doi:10.3766/jaaa. 16.7.7.

[11] Zhang, X. Polar plots of a directional microphone with realworld sounds and its spectrum distortion, EPH -- International Journal of Science and Engineering, 2017, 3(7), 1-11.

[12] Thomapson, C. S., LoPresti, L. J., Ring, M. E., Nepomuceno, G. $\mathrm{H}$., et al. Noise in miniature microphones, J. Acoust Soc. Am. 2002, 111(2), 861-866. Doi:10.1121/1.428971.

[13] Ryoo, K., Chilukuri, M., Jung, S. A low power and low noise pre-amplifier circuit for hearing aid devices, Circuits and Systems Conference (DCAS) IEEE, Texas, 2016. Doi: $10.1109 /$ dcas.2016.7791128.

[14] Directional Microphones, Sonion. $\mathrm{http} / /$ www.sonion.com/wp/products/hearing- instrumentcomponents/microphones/directional-microphones/.

[15] Voice Samples-LAMP Words for Life. https://aacapps.com/lamp/voices.

[16] Mahendru, H. C., Khanna, R. Spectral analysis of various noise signals affecting mobile speech communication, International Journal of Scientific \& Engineering Research, 2014, 5, 10871093.

[17] Fontan, L., Tardieu, J., Gaillard, P., Woisard, V., Ruiz, R. Relationship between speech intelligibility and speech comprehension in babble noise, J. Speech Lang Hear Res., 2015, 58, 977-986. Doi: 10.1044/2015 jslhr-h-13- 0335.

[18] Venema, H. T. Compression for Clinicians, 2nd Ed., Thomson Delmar Learning, 2006. 


\section{Biography}

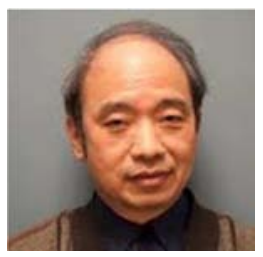

Xubao Zhang received his doctorate in electronics from Xi'an Electronic Science and Technology University in China and was a postdoctoral fellow at McMaster University in Canada. He has been interested in hearing aid technology strategies and performance evaluation. He worked as an EA and EMC engineer with Sonova Unitron, also with Oticon Canada. And he worked as Associate Professor at EE department of the Xidian University for radar signal processing research. He is the author of one book and more than 40 articles. 